Supporting information for:

\title{
Studies Directed Toward the Construction of the Polypropionate Fragment of Superstolide A
}

\author{
Joan G. Solsona, Pedro Romea, ${ }^{*}$ Fèlix Urpí*
}

\section{Experimental procedures and characterization data for compounds $2,4,5$, and $8-17$.}

Melting points were taken on an Electrothermal apparatus and have not been corrected. Specific rotations were determined at $20{ }^{\circ} \mathrm{C}$ on a Perkin-Elmer $241 \mathrm{MC}$ polarimeter. IR spectra were recorded on either a Perkin-Elmer 681 or a Nicolet 510 FT spectrometer and only the more representative frequencies $\left(\mathrm{cm}^{-1}\right)$ are reported. ${ }^{1} \mathrm{H}$ NMR $(300 \mathrm{MHz})$ and

${ }^{13} \mathrm{C}$ NMR $(75.4 \mathrm{MHz})$ spectra were recorded on a Varian Unity Plus 300 spectrometer; ${ }^{1} \mathrm{H}$ NMR $(400 \mathrm{MHz})$ and ${ }^{13} \mathrm{C}$ NMR (100.6 MHz) spectra were recorded on a Varian Mercury-400 spectrometer; ${ }^{1} \mathrm{H}$ NMR (500 MHz) spectra were recorded in a Varian Inova-500 spectrometer. Chemical shifts $(\delta)$ are quoted in ppm and referenced to internal TMS for ${ }^{1} \mathrm{H}$ NMR and $\mathrm{CDCl}_{3}(\delta 77.0)$ or $\mathrm{CD}_{3} \mathrm{OD}(\delta 49.0)$ for ${ }^{13} \mathrm{C}$ NMR; coupling constants $(J)$ are quoted in $\mathrm{Hz}$; data are reported as follows: s, singlet; $\mathrm{d}$, doublet; $\mathrm{t}$, triplet; q, quartet; $\mathrm{m}$, multiplet; br, broad; where appropriate, 2D techniques were also used to assist in structure elucidation. High resolution mass spectra (HRMS) were obtained from the Centro de Apoio Cientifico Tecnoloxico a Investigacion (C.A.C.T.I.), Universidad de Vigo. HPLC analyses were performed on a Shimadzu Liquid Chromatograph (Tracer Spehrisorb S3W column, $4.6 \mathrm{~mm} \times 25 \mathrm{~cm}, 3 \mu \mathrm{m}$ silica gel, $0.9 \mathrm{~mL} \min ^{-1}$ ). Flash chromatography was performed on SDS silica gel $60(35-70 \mu \mathrm{m})$. Analytical thin-layer chromatography was carried out on Merck Kieselgel $60 \mathrm{~F}_{254}$ plates.

All reactions were conducted in oven-dried glassware under inert atmosphere of dry argon or nitrogen with anhydrous solvents. The solvents and reagents were purified and dried according to standard procedures. 


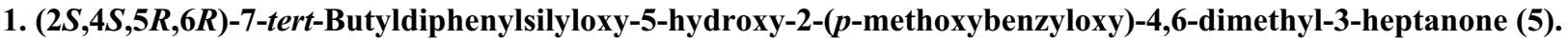

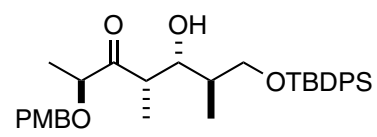

Freshly distilled $\mathrm{Ti}(i-\mathrm{PrO})_{4}(196 \mu \mathrm{L}, 0.66 \mathrm{mmol})$ was added dropwise to a solution of $\mathrm{TiCl}_{4}(218 \mu \mathrm{L}, 1.98 \mathrm{mmol})$ in $\mathrm{CH}_{2} \mathrm{Cl}_{2}(2.3 \mathrm{~mL})$ at $0{ }^{\circ} \mathrm{C}$ under $\mathrm{N}_{2}$ and the yellow solution was stirred for $10 \mathrm{~min}$ at $0{ }^{\circ} \mathrm{C}$ and $10 \mathrm{~min}$ at $\mathrm{rt}$. It was then diluted with $\mathrm{CH}_{2} \mathrm{Cl}_{2}(2.3 \mathrm{~mL})$ and the resulting colorless solution was added dropwise (it was rinsed with $2 \times 1.0 \mathrm{~mL}$ ) for $10-15 \mathrm{~min}$ to a solution of $6(535 \mathrm{mg}, 2.4 \mathrm{mmol})$ in $\mathrm{CH}_{2} \mathrm{Cl}_{2}(4.3 \mathrm{~mL})$ at $-90{ }^{\circ} \mathrm{C}$ under $\mathrm{N}_{2}$, followed by $i-\mathrm{Pr}_{2} \mathrm{EtN}(0.46 \mathrm{~mL}$, $2.6 \mathrm{mmol})$. The resulting red solution was stirred for $1.5 \mathrm{~h}$ at $-78{ }^{\circ} \mathrm{C}$ and a solution of $7\left(943 \mathrm{mg}, 2.9 \mathrm{mmol}^{2} \mathrm{in} \mathrm{CH}_{2} \mathrm{Cl}_{2}\right.$ $(1.0 \mathrm{~mL})$ was added slowly (it was rinsed with $2 \times 0.5 \mathrm{~mL})$.

After $2 \mathrm{~h}$ at $-78^{\circ} \mathrm{C}$, the reaction mixture was quenched by the addition of saturated $\mathrm{NH}_{4} \mathrm{Cl}(12 \mathrm{~mL})$ and was vigorously stirred at rt. The mixture was partitioned with $\mathrm{Et}_{2} \mathrm{O}(450 \mathrm{~mL})$ and $\mathrm{H}_{2} \mathrm{O}(120 \mathrm{~mL})$ and the organic layer was washed with saturated $\mathrm{NaHCO}_{3}(120 \mathrm{~mL})$, and brine $(120 \mathrm{~mL})$. The aqueous layers were further extracted with $\mathrm{Et}_{2} \mathrm{O}(200 \mathrm{~mL})$ and the combined organic extracts were dried $\left(\mathrm{MgSO}_{4}\right)$, filtered, and concentrated. The resulting oil was analyzed by HPLC and purified by flash column chromatography (hexanes/EtOAc 90:10), affording $1.079 \mathrm{~g}(82 \%)$ of 5 .

Colorless oil. $\boldsymbol{R}_{\boldsymbol{f}}$ (hexanes/EtOAc 85:15) =0.25. HPLC (hexanes $/ i-\mathrm{PrOH}$ 99.5:0.5) $\boldsymbol{t}_{\mathbf{R}}=13.4 \mathrm{~min} .[\boldsymbol{\alpha}]_{\mathbf{D}}=-11.5(c=1.10$, $\mathrm{CHCl}_{3}$ ). IR (film): $v 3504$ (br), 3072, 2962, 2935, 1713, 1613, 1515, 1250, 1113, $1036 \mathrm{~cm}^{-1} .{ }^{1} \mathbf{H ~ N M R}(500 \mathrm{MHz}$, $\left.\mathrm{CDCl}_{3}\right) \delta$ 7.70-7.60 (4H, m, ArH), 7.45-7.35 (6H, m, ArH), 7.28-7.22 (2H, m, ArH), 6.88-6.82 (2H, m, ArH), 4.49 (1H, $\left.\mathrm{d}, J=11.5, \mathrm{OC}_{\mathrm{x}} \mathrm{H}_{\mathrm{y}} \mathrm{Ar}\right), 4.45\left(1 \mathrm{H}, \mathrm{d}, J=11.5, \mathrm{OCH}_{\mathrm{x}} \underline{\mathrm{H}}_{\mathrm{y}} \mathrm{Ar}\right), 4.08(1 \mathrm{H}, \mathrm{q}, J=6.9, \mathrm{C} \underline{\mathrm{HOPMB}}), 4.00-3.95(1 \mathrm{H}, \mathrm{m}, \mathrm{C} \underline{\mathrm{HOH}})$, 3.80-3.60 (1H, m, $\left.\underline{\mathrm{C}}_{\mathrm{x}} \mathrm{H}_{\mathrm{y}} \mathrm{OSi}\right), 3.78\left(3 \mathrm{H}, \mathrm{s}, \mathrm{OCH}_{3}\right), 3.67\left(1 \mathrm{H}, \mathrm{dd}, J=10.1, J=6.1, \mathrm{CH}_{\mathrm{x}} \underline{\mathrm{H}}_{\mathrm{y}} \mathrm{OSi}\right), 3.10(1 \mathrm{H}, \mathrm{qd}, J=7.0, J=$ 3.2, $\left.\mathrm{COC}^{\mathrm{HCH}}{ }_{3}\right), 1.80-1.70\left(1 \mathrm{H}, \mathrm{m}, \mathrm{CHCH}_{2} \mathrm{OSi}\right), 1.36\left(3 \mathrm{H}, \mathrm{d}, J=6.9, \mathrm{C}_{3} \mathrm{CHOPMB}\right), 1.09$ (3H, d, $\left.J=7.0, \mathrm{COCHC}_{3}\right)$, $1.04\left(9 \mathrm{H}, \mathrm{s}, \mathrm{C}\left(\mathrm{CH}_{3}\right)_{3}\right), 0.85\left(3 \mathrm{H}, \mathrm{d}, J=6.9, \mathrm{C}_{3} \mathrm{CHCH}_{2} \mathrm{OSi}\right) .{ }^{13} \mathbf{C} \mathbf{N M R}\left(75.4 \mathrm{MHz}, \mathrm{CDCl}_{3}\right) \delta 215.4(\mathrm{C}), 159.3(\mathrm{C}), 135.6$ $(\mathrm{CH}), 133.1(\mathrm{C}), 133.0(\mathrm{C}), 129.8(\mathrm{CH}), 129.4(\mathrm{CH}), 127.7(\mathrm{CH}), 113.8(\mathrm{CH}), 79.0(\mathrm{CH}), 74.3(\mathrm{CH}), 71.4\left(\mathrm{CH}_{2}\right), 68.0$ $\left(\mathrm{CH}_{2}\right), 55.3\left(\mathrm{CH}_{3}\right), 44.1(\mathrm{CH}), 37.6(\mathrm{CH}), 26.8\left(\mathrm{CH}_{3}\right), 19.2(\mathrm{C}), 17.4\left(\mathrm{CH}_{3}\right), 13.6\left(\mathrm{CH}_{3}\right), 8.7\left(\mathrm{CH}_{3}\right) . \mathbf{H R M S}(+\mathrm{FAB}): m / z$ calcd. for $\mathrm{C}_{33} \mathrm{H}_{45} \mathrm{O}_{5} \mathrm{Si}[\mathrm{M}+\mathrm{H}]^{+}:$549.3036. Found: 549.3029. 
Supporting information for Studies Directed Toward the Construction of the Polypropionate Fragment of Superstolide A Joan G. Solsona, Pedro Romea, Fèlix Urpí

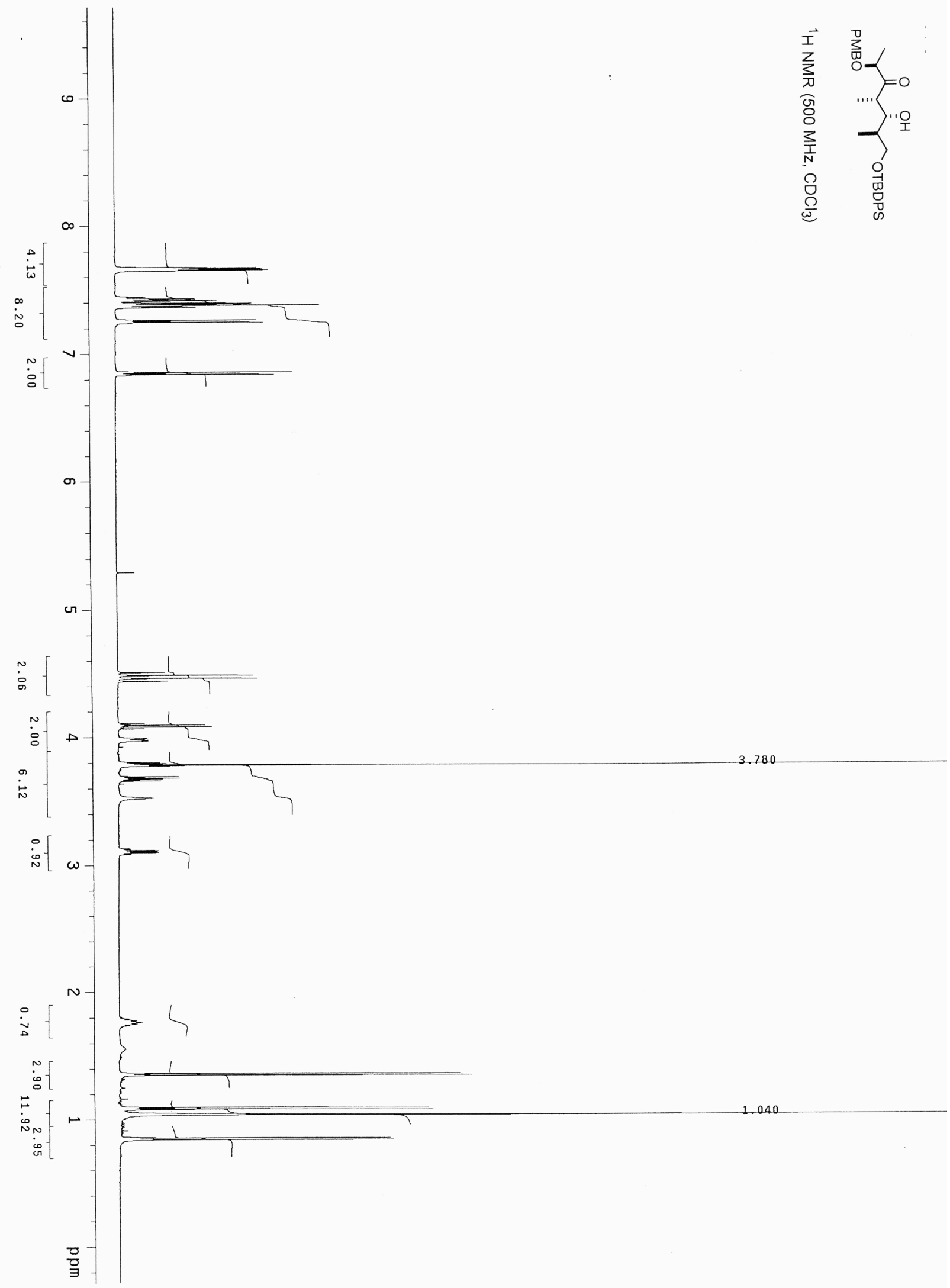


Supporting information for Studies Directed Toward the Construction of the Polypropionate Fragment of Superstolide A Joan G. Solsona, Pedro Romea, Fèlix Urpí

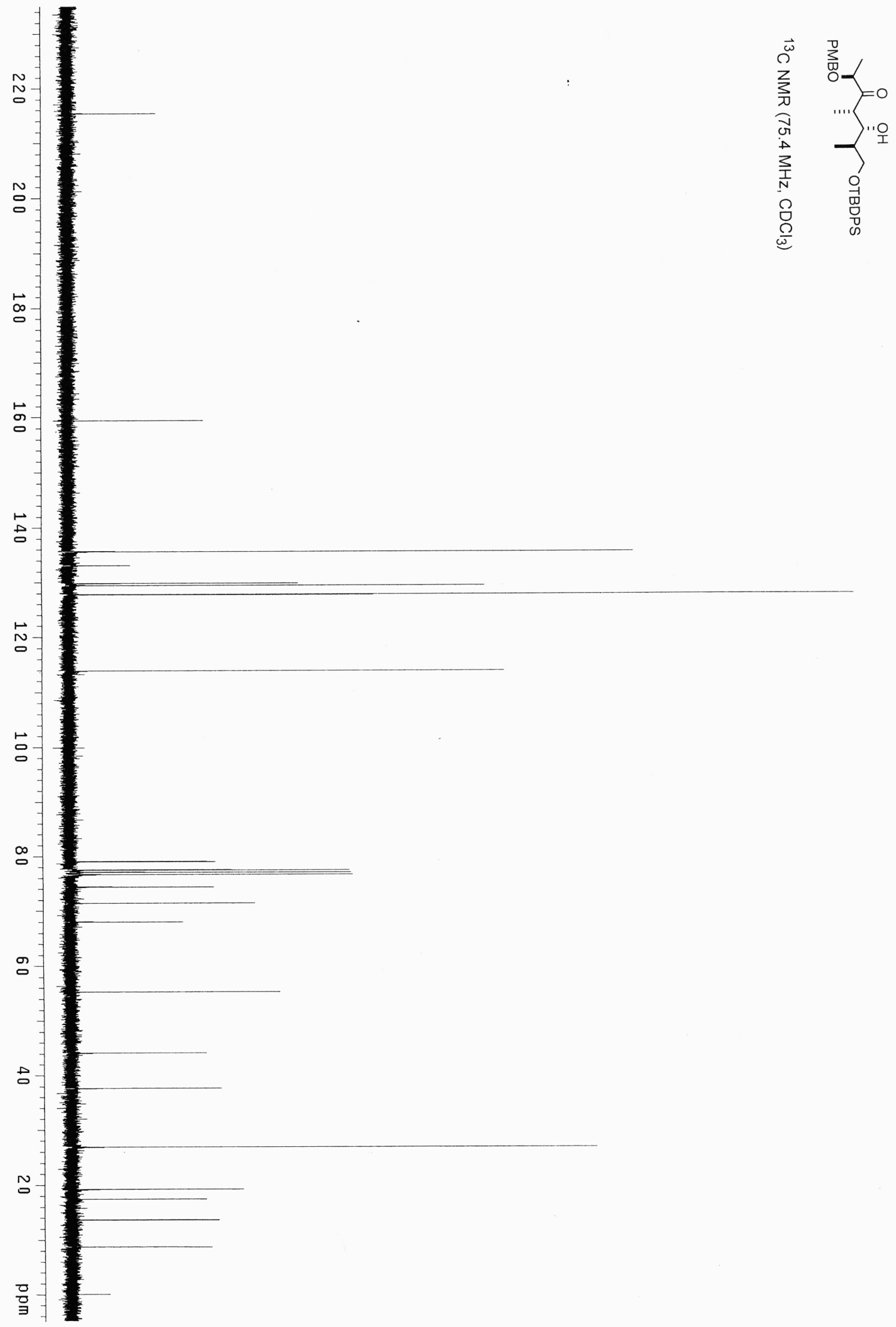




\section{2. (2R,3R,4S,5S,6S)-1-tert-Butyldiphenylsilyloxy-6-(4-methoxybenzyloxy)-2,4-dimethyl-3,5-heptandiol (8).}<smiles>CC(C)O[Pb](C)(C)C</smiles>

Glacial $\mathrm{AcOH}(8.1 \mathrm{~mL})$ was added to a solution of $\left(\mathrm{Me}_{4} \mathrm{~N}\right) \mathrm{HB}(\mathrm{OAc})_{3}(3.795 \mathrm{~g}, 13.9 \mathrm{mmol})$ in $\mathrm{CH}_{3} \mathrm{CN}(8.1 \mathrm{~mL})$ and the resulting mixture was stirred at $\mathrm{rt}$ for $1 \mathrm{~h}$. It was cooled to $-40{ }^{\circ} \mathrm{C}$ and a solution of $5(873 \mathrm{mg}, 1.6 \mathrm{mmol}) \mathrm{in} \mathrm{CH}_{3} \mathrm{CN}(3.2$ $\mathrm{mL}$ ) was added dropwise (it was rinsed with $2 \times 1.5 \mathrm{~mL}$ of $\mathrm{CH}_{3} \mathrm{CN}$ ). The reaction mixture was stirred at $-40{ }^{\circ} \mathrm{C}$ for $5 \mathrm{~h}$, kept overnight in the fridge $\left(-25^{\circ} \mathrm{C}\right)$ and stirred for $1 \mathrm{~h}$ at $0{ }^{\circ} \mathrm{C}$.

The reaction was carefully quenched by addition of a $0.5 \mathrm{M}$ aqueous solution of sodium potassium tartrate $(27 \mathrm{~mL})$ and stirred for $1 \mathrm{~h}$ at $\mathrm{rt}$. The mixture was diluted with $\mathrm{CH}_{2} \mathrm{Cl}_{2}(40 \mathrm{~mL})$ and washed with saturated $\mathrm{NaHCO}_{3}(40 \mathrm{~mL})$. The aqueous layer was extracted with $\mathrm{CH}_{2} \mathrm{Cl}_{2}(6 \times 40 \mathrm{~mL})$ and the combined organic extracts were washed with saturated $\mathrm{NaHCO}_{3}(3 \times 40 \mathrm{~mL})$, dried $\left(\mathrm{MgSO}_{4}\right)$, filtered, and concentrated. The resulting oil was analyzed by HPLC (hexanes/iPrOH 99:1), which showed the presence of two diols in a 94:6 dr. Purification through a flash column chromatography (from hexanes/EtOAc 95:5 to hexanes/EtOAc 80:20) afforded $806 \mathrm{mg}(92 \%)$ of diastereomerically pure 8.

Colorless wax. $\boldsymbol{R}_{\boldsymbol{f}}$ (hexanes/EtOAc 85:15) =0.10. HPLC (hexanes/i-PrOH 99:1) $\boldsymbol{t}_{\mathbf{R}}=12.4 \mathrm{~min} .[\boldsymbol{\alpha}]_{\mathbf{D}}=+8.4(c=1.36$, $\mathrm{CHCl}_{3}$ ). IR (film): v 3490 (br), 3072, 2964, 2933, 1613, 1515, 1250, 1113, $1038 \mathrm{~cm}^{-1} .{ }^{1} \mathbf{H} \mathbf{~ N M R}\left(400 \mathrm{MHz}, \mathrm{CDCl}_{3}\right)$ S 7.72-7.64 (4H, m, ArH), 7.46-7.34 (6H, m, ArH), 7.28-7.22 (2H, m, ArH), 6.90-6.84 (2H, m, ArH), 4.63 (1H, d, J= 11.3, OC $\left.\underline{\mathrm{H}}_{\mathrm{x}} \mathrm{H}_{\mathrm{y}} \mathrm{Ar}\right), 4.38\left(1 \mathrm{H}, \mathrm{d}, J=11.3, \mathrm{OCH}_{\mathrm{x}} \underline{\mathrm{H}}_{\mathrm{y}} \mathrm{Ar}\right), 3.91\left(1 \mathrm{H}, \mathrm{d}, J=9.6, \mathrm{C}^{\mathrm{HOHCHCH}} \mathrm{OSi}\right), 3.82(1 \mathrm{H}, \mathrm{s}, \mathrm{OH}), 3.79$ $\left(3 \mathrm{H}, \mathrm{s}, \mathrm{OCH}_{3}\right), 3.75\left(2 \mathrm{H}, \mathrm{d}, J=5.4, \mathrm{CH}_{2} \mathrm{OSi}\right), 3.67(1 \mathrm{H}$, quintet, $J=6.1$, $\mathrm{CH}(\mathrm{OPMB}) \mathrm{C} \underline{\mathrm{HOH}}), 2.84(1 \mathrm{H}, \mathrm{d}, J=5.2, \mathrm{OH}), 1.90-1.75\left(2 \mathrm{H}, \mathrm{m}, 2 \times \mathrm{CHCH}_{3}\right), 1.22\left(3 \mathrm{H}, \mathrm{d}, J=6.1, \underline{\mathrm{H}}_{3} \mathrm{CHOPMB}\right)$, $1.06\left(9 \mathrm{H}, \mathrm{s}, \mathrm{C}\left(\mathrm{CH}_{3}\right)_{3}\right), 0.94\left(3 \mathrm{H}, \mathrm{d}, J=7.0, \mathrm{CHCH}_{3}\right), 0.78\left(3 \mathrm{H}, \mathrm{d}, J=6.9, \mathrm{CHCH}_{3}\right) .{ }^{13} \mathbf{C ~ N M R}(100.6 \mathrm{MHz}, \mathrm{CDCl}) \delta$ $159.3(\mathrm{C}), 135.6(\mathrm{CH}), 135.5(\mathrm{CH}), 133.4(\mathrm{C}), 133.3(\mathrm{C}), 130.5(\mathrm{C}), 129.6(\mathrm{CH}), 129.4(\mathrm{CH}), 127.7(\mathrm{CH}), 113.8(\mathrm{CH})$, $79.1(\mathrm{CH}), 75.4(\mathrm{CH}), 73.6(\mathrm{CH}), 70.7\left(\mathrm{CH}_{2}\right), 68.2\left(\mathrm{CH}_{2}\right), 55.2\left(\mathrm{CH}_{3}\right), 38.0(\mathrm{CH}), 35.3(\mathrm{CH}), 26.8\left(\mathrm{CH}_{3}\right), 19.2(\mathrm{C}), 15.9$ $\left(\mathrm{CH}_{3}\right), 13.2\left(\mathrm{CH}_{3}\right), 10.1\left(\mathrm{CH}_{3}\right)$. HRMS (+FAB): $m / z$ calcd. for $\mathrm{C}_{33} \mathrm{H}_{47} \mathrm{O}_{5} \mathrm{Si}[\mathrm{M}+\mathrm{H}]^{+}:$551.3193. Found: 551.3169 . 


\section{3. (2R,3R,4S,5S,6S)-1-tert-Butyldiphenylsilyloxy-3,5-isopropylidenedioxy-6-(4-methoxybenzyloxy)-}

\section{2,4-dimethylheptane (9).}

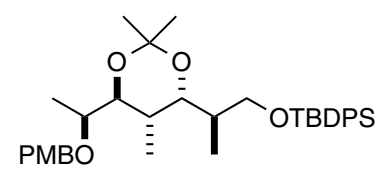

A solution of $8(742 \mathrm{mg}, 1.35 \mathrm{mmol})$ and a catalytic amount of PPTS in 1:1 $\mathrm{CH}_{2} \mathrm{Cl}_{2} / 2$,2-dimethoxypropane (30 mL) was stirred for one day at $\mathrm{rt}$ under $\mathrm{N}_{2}$. It was concentrated in vacuo and the residue was purified by flash column chromatography (hexanes/EtOAc 97:3), which afforded $732 \mathrm{mg}(92 \%)$ of 9.

Colorless wax. $\boldsymbol{R}_{\boldsymbol{f}}$ (hexanes/EtOAc 95:5) $=0.20 .[\alpha]_{\mathbf{D}}=-31.9\left(c=1.18, \mathrm{CHCl}_{3}\right)$. IR (film): v 3072, 2966, 2935, 1613, 1515, 1248, 1225, $1113 \mathrm{~cm}^{-1} .{ }^{1} \mathbf{H}$ NMR (500 MHz, CDCl $) \delta 7.70-7.62(4 \mathrm{H}, \mathrm{m}, \mathrm{ArH}), 7.44-7.32$ (6H, m, ArH), 7.28-7.24 (2H, m, ArH), 6.88-6.84 (2H, m, ArH), $4.61\left(1 \mathrm{H}, \mathrm{d}, J=11.8, \mathrm{OC}_{\mathrm{x}} \mathrm{H}_{\mathrm{y}} \mathrm{Ar}\right), 4.46\left(1 \mathrm{H}, \mathrm{d}, J=11.8, \mathrm{OCH}_{\mathrm{x}} \underline{\mathrm{H}}_{\mathrm{y}} \mathrm{Ar}\right)$, $3.79\left(3 \mathrm{H}, \mathrm{s}, \mathrm{OCH}_{3}\right), 3.73-3.64\left(3 \mathrm{H}, \mathrm{m}, \mathrm{OC}^{\mathrm{HCHCH}}{ }_{2} \mathrm{OSi}\right), 3.56(1 \mathrm{H}, \mathrm{qd}, J=6.4, J=4.1$, C $7.0, J=4.1, \mathrm{CH}(\mathrm{OPMB}) \mathrm{CHO}), 1.98(1 \mathrm{H}$, quintetd, $J=7.0, J=4.1, \mathrm{OCHC} \underline{H C H O}), 1.73-1.64\left(1 \mathrm{H}, \mathrm{m}, \mathrm{CHCH}_{2} \mathrm{OSi}\right), 1.28$ $\left(3 \mathrm{H}, \mathrm{s}, \mathrm{CH}_{3} \mathrm{CO}_{2}\right), 1.24\left(3 \mathrm{H}, \mathrm{s}, \mathrm{CH}_{3} \mathrm{CO}_{2}\right), 1.20\left(3 \mathrm{H}, \mathrm{d}, J=6.4, \mathrm{CH}_{3} \mathrm{CHOPMB}\right), 1.06\left(9 \mathrm{H}, \mathrm{s}, \mathrm{C}_{\left(\mathrm{CH}_{3}\right)}\right), 0.91(3 \mathrm{H}, \mathrm{d}, J=7.0$, $\left.\mathrm{OCHCH}\left(\mathrm{CH}_{3}\right) \mathrm{CHO}\right), 0.80\left(3 \mathrm{H}, \mathrm{d}, J=6.7, \mathrm{CH}\left(\mathrm{CH}_{3}\right) \mathrm{CH}_{2} \mathrm{OSi}\right) .{ }^{13} \mathbf{C} \mathbf{N M R}\left(75.4 \mathrm{MHz}, \mathrm{CDCl}_{3}\right) \delta 159.1(\mathrm{C}), 135.8(\mathrm{CH})$, $135.7(\mathrm{CH}), 134.1(\mathrm{C}), 134.0(\mathrm{C}), 131.0(\mathrm{C}), 129.4(\mathrm{CH}), 129.4(\mathrm{CH}), 129.3(\mathrm{CH}), 127.5(\mathrm{CH}), 127.4(\mathrm{CH}), 113.7(\mathrm{CH})$, $100.6(\mathrm{C}), 77.5(\mathrm{CH}), 74.6(\mathrm{CH}), 71.0\left(\mathrm{CH}_{2}\right), 69.8(\mathrm{CH}), 65.2\left(\mathrm{CH}_{2}\right), 55.3\left(\mathrm{CH}_{3}\right), 35.5(\mathrm{CH}), 33.0(\mathrm{CH}), 27.0\left(\mathrm{CH}_{3}\right), 25.5$ $\left(\mathrm{CH}_{3}\right), 23.5\left(\mathrm{CH}_{3}\right), 19.4(\mathrm{C}), 15.3\left(\mathrm{CH}_{3}\right), 13.1\left(\mathrm{CH}_{3}\right), 12.3\left(\mathrm{CH}_{3}\right)$. HRMS $(+\mathrm{FAB}): \mathrm{m} / z$ calcd. for $\mathrm{C}_{36} \mathrm{H}_{51} \mathrm{O}_{5} \mathrm{Si}[\mathrm{M}+\mathrm{H}]^{+}$: 591.3506. Found: 591.3503 . 


\section{Protected azido poliol 4.}<smiles>CC(CO[Pb])[C@H]1OC(C)(C)O[C@@H]([C@H](C)N)[C@@H]1C</smiles>

DDQ (186 mg, $0.82 \mathrm{mmol})$ was added to a mixture of 9 (373 mg, $0.63 \mathrm{mmol})$ in 10:1 $\mathrm{CH}_{2} \mathrm{Cl}_{2} /$ phosphate pH 7 buffer (16.5 $\mathrm{mL})$ at $0{ }^{\circ} \mathrm{C}$. The reaction mixture was vigorously stirred for $2 \mathrm{~h}$ at $0{ }^{\circ} \mathrm{C}$ and partitioned between $\mathrm{CH}_{2} \mathrm{Cl}_{2}(200 \mathrm{~mL})$ and saturated $\mathrm{NaHCO}_{3}(120 \mathrm{~mL})$. The aqueous layer was extracted with $\mathrm{CH}_{2} \mathrm{Cl}_{2}(2 \times 150 \mathrm{~mL})$ and the combined organic extracts were washed with saturated $\mathrm{NaHCO}_{3}(120 \mathrm{~mL})$, dried $\left(\mathrm{MgSO}_{4}\right)$, filtered, and concentrated. The resulting oil was eluted through a pad of silica gel (hexanes/EtOAc 85:15) and the colorless oil (396 mg) remaining after evaporation of the volatiles was taken on to the next reaction without further purification.

It was dissolved in $\mathrm{CH}_{2} \mathrm{Cl}_{2}(8.8 \mathrm{~mL})$, cooled to $0{ }^{\circ} \mathrm{C}$ under $\mathrm{N}_{2}$, and $\mathrm{Et}_{3} \mathrm{~N}$ (132 $\left.\mu \mathrm{L}, 0.95 \mathrm{mmol}\right)$ and $\mathrm{MsCl}(59 \mu \mathrm{L}, 0.76$ mmol) were added dropwise. The reaction was stirred for $4 \mathrm{~h}$ at $0{ }^{\circ} \mathrm{C}$, diluted with $\mathrm{CH}_{2} \mathrm{Cl}_{2}(40 \mathrm{~mL})$, and washed with $\mathrm{H}_{2} \mathrm{O}$ $(50 \mathrm{~mL})$, saturated $\mathrm{NaHCO}_{3}(50 \mathrm{~mL})$, and $\mathrm{H}_{2} \mathrm{O}(50 \mathrm{~mL})$. The organic layer was dried $\left(\mathrm{Na}_{2} \mathrm{SO}_{4}\right)$, filtered, and concentrated. The resulting oil was taken on to the next reaction without further purification.

It was diluted with DMF (2.5 mL) and $\mathrm{NaN}_{3}(305 \mathrm{mg}, 4.69 \mathrm{mmol})$ was added. The resulting suspension was stirred for 22 $\mathrm{h}$ at $70{ }^{\circ} \mathrm{C}$. The mixture was diluted with $\mathrm{Et}_{2} \mathrm{O}(50 \mathrm{~mL})$ and washed with $\mathrm{H}_{2} \mathrm{O}(3 \times 15 \mathrm{~mL})$, and brine $(15 \mathrm{~mL})$. The organic layer was dried $\left(\mathrm{MgSO}_{4}\right)$, filtered, and concentrated. The resulting oil was purified by flash column chromatography (hexanes/EtOAc 97:3), affording $250 \mathrm{mg}(80 \%)$ of 4.

Colorless oil. $\boldsymbol{R}_{\boldsymbol{f}}$ (hexanes/EtOAc 97:3) $=0.40$. $[\boldsymbol{\alpha}]_{\mathbf{D}}=-24.9\left(c=1.11, \mathrm{CHCl}_{3}\right)$. IR (film): v 3074, 2935, 2101, 1474, 1428, 1380, 1225, 1113, $1026 \mathrm{~cm}^{-1} .{ }^{1} \mathbf{H}$ NMR (400 MHz, $\left.\mathrm{CDCl}_{3}\right) \delta 7.70-7.63$ (4H, m, ArH), 7.45-7.33 (6H, m, ArH), 3.77-3.65 (3H, m, OCㅌCHCH$\left.\underline{H}_{2} \mathrm{OSi}\right), 3.50\left(1 \mathrm{H}, \mathrm{qd}, J=6.7, J=4.2, \mathrm{CHN}_{3}\right), 3.35\left(1 \mathrm{H}, \mathrm{dd}, J=6.6, J=4.2, \mathrm{CH}\left(\mathrm{N}_{3}\right) \mathrm{CHO}\right)$, $1.87(1 \mathrm{H}$, quintetd, $J=6.6, J=3.9$, OCHCㅡㅐ $\left.=6.7, \mathrm{CH}_{3} \mathrm{CHN}_{3}\right), 1.24\left(3 \mathrm{H}, \mathrm{s}, \mathrm{CH}_{3} \mathrm{CO}_{2}\right), 1.07\left(9 \mathrm{H}, \mathrm{s}, \mathrm{C}\left(\mathrm{CH}_{3}\right)_{3}\right), 0.94\left(3 \mathrm{H}, \mathrm{d}, J=6.7, \mathrm{CH}\left(\mathrm{CH}_{3}\right) \mathrm{CH}_{2} \mathrm{OSi}\right), 0.92(3 \mathrm{H}, \mathrm{d}, J=$ 6.6, $\left.\mathrm{OCHCH}\left(\mathrm{CH}_{3}\right) \mathrm{CHO}\right) .{ }^{13} \mathrm{C}$ NMR $\left(100.6 \mathrm{MHz}, \mathrm{CDCl}_{3}\right) \delta 135.8(\mathrm{CH}), 135.7(\mathrm{CH}), 134.0(\mathrm{C}), 133.9(\mathrm{C}), 129.5(\mathrm{CH})$, 129.4 (CH), $127.5(\mathrm{CH}), 100.7(\mathrm{C}), 78.1(\mathrm{CH}), 69.5(\mathrm{CH}), 65.0\left(\mathrm{CH}_{2}\right), 59.6(\mathrm{CH}), 35.4(\mathrm{CH}), 34.0(\mathrm{CH}), 27.0\left(\mathrm{CH}_{3}\right), 25.3$ $\left(\mathrm{CH}_{3}\right), 23.6\left(\mathrm{CH}_{3}\right), 19.4(\mathrm{C}), 14.6\left(\mathrm{CH}_{3}\right), 13.0\left(\mathrm{CH}_{3}\right), 12.7\left(\mathrm{CH}_{3}\right)$. HRMS $(+\mathrm{FAB}): \mathrm{m} / z$ calcd. for $\mathrm{C}_{28} \mathrm{H}_{42} \mathrm{NO}_{3} \mathrm{Si}[\mathrm{M}+\mathrm{H}]^{+}$: 496.2995. Found: 496.2995. 
Supporting information for Studies Directed Toward the Construction of the Polypropionate Fragment of Superstolide A Joan G. Solsona, Pedro Romea, Fèlix Urpí

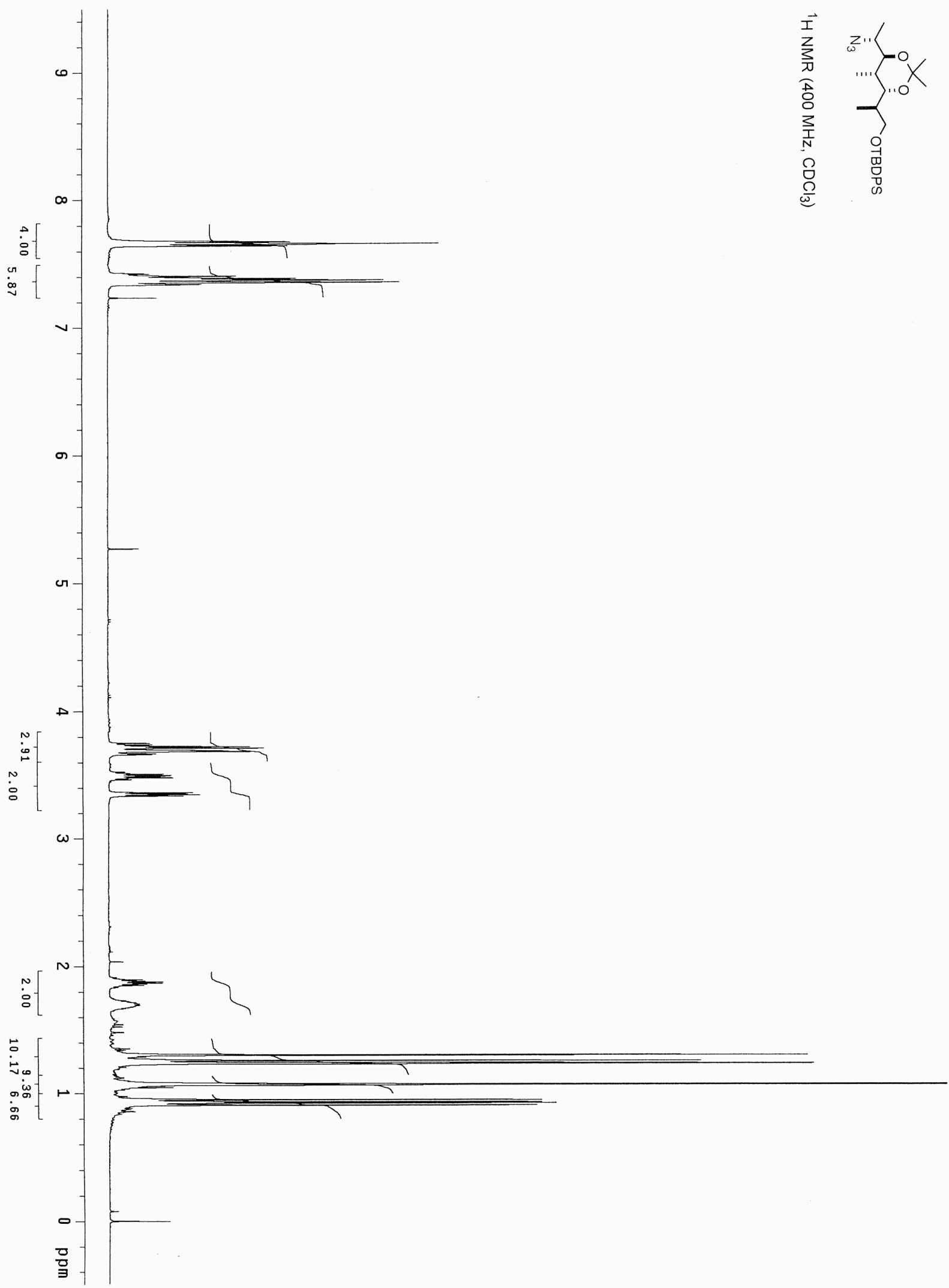


Supporting information for Studies Directed Toward the Construction of the Polypropionate Fragment of Superstolide A Joan G. Solsona, Pedro Romea, Fèlix Urpí

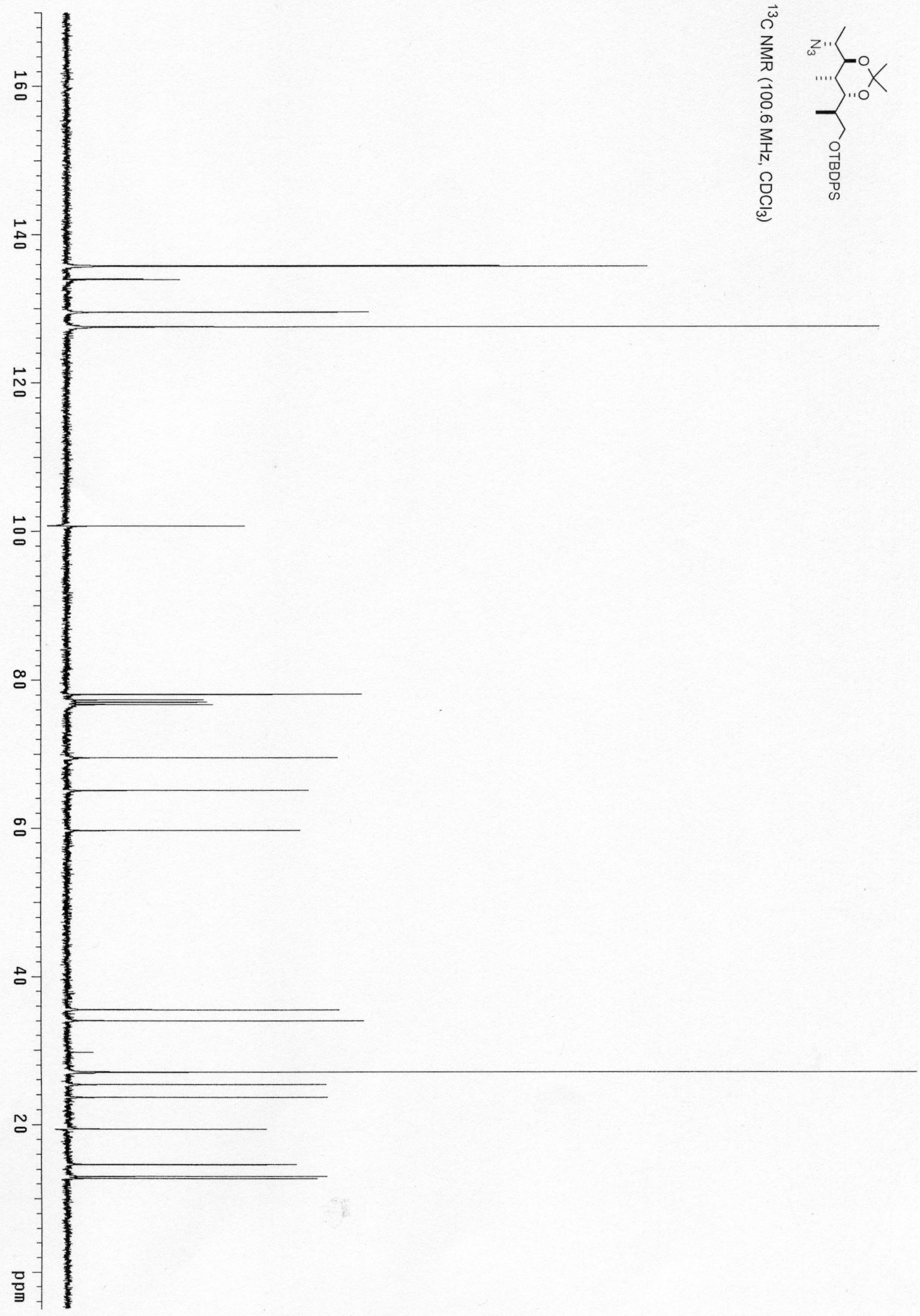




\section{Acetamide derivative 10.}<smiles>CC(=O)N[C@@H](C)C1OC(C)(C)OC([C@H](C)C(C)CO[Pb])[C@H]1C</smiles>

A $1 \mathrm{M}$ solution of $\mathrm{Me}_{3} \mathrm{P}$ in toluene $(0.18 \mathrm{~mL}, 0.18 \mathrm{mmol})$ was added dropwise to a solution of $4(69.6 \mathrm{mg}, 0.14 \mathrm{mmol})$ and water $(10 \mu \mathrm{L})$ in THF $(1.5 \mathrm{~mL})$ under $\mathrm{N}_{2}$. The reaction mixture was stirred overnight at rt and concentrated. Water was removed by adding toluene $(3 \times 4 \mathrm{~mL})$ and concentrating in vacuo.

The residue was dissolved in $\mathrm{CH}_{2} \mathrm{Cl}_{2}(1.5 \mathrm{~mL})$ and $\mathrm{Et}_{3} \mathrm{~N}(215 \mu \mathrm{L}, 1.54 \mathrm{mmol})$ and $\mathrm{Ac}_{2} \mathrm{O}(23 \mu \mathrm{L}, 0.24 \mathrm{mmol})$ were successively added. The reaction mixture was stirred for $1 \mathrm{~h}$ at $\mathrm{rt}$. The solvent was removed and the resulting oil was purified by flash column chromatography (hexanes/EtOAc 50:50), which provided $63.2 \mathrm{mg}$ (88\%) of $\mathbf{1 0}$.

Viscous oil. $\boldsymbol{R}_{\boldsymbol{f}}$ (hexanes/EtOAc 50:50) =0.40. $[\boldsymbol{\alpha}]_{\mathbf{D}}=-2.1\left(c=1.03, \mathrm{CHCl}_{3}\right)$. IR (film): v 3284 (br), 2966, 2935, 1654, 1559, 1542, 1459, 1225, 1113, $1023 \mathrm{~cm}^{-1} .{ }^{1} \mathbf{H}$ NMR (400 MHz, $\left.\mathrm{CDCl}_{3}\right) \delta$ 7.70-7.60 (4H, m, ArH), 7.45-7.30 (6H, m, $\mathrm{ArH}), 5.74(1 \mathrm{H}, \mathrm{d}, J=8.8, \mathrm{NH}), 4.14-4.00(1 \mathrm{H}, \mathrm{m}, \mathrm{C} \underline{\mathrm{HNH}}), 3.71\left(1 \mathrm{H}, \mathrm{dd}, J=9.7, J=5.1, \mathrm{C}_{\mathrm{x}} \mathrm{H}_{\mathrm{y}} \mathrm{OTBDPS}\right), 3.67(1 \mathrm{H}$, $\left.\mathrm{dd}, J=9.7, J=3.1, \mathrm{CH}_{\mathrm{x}} \underline{\mathrm{H}}_{\mathrm{y}} \mathrm{OTBDPS}\right), 3.62\left(1 \mathrm{H}, \mathrm{dd}, J=10.8, J=4.2, \mathrm{C}_{\mathbf{H O C H C H}} \mathrm{OSi}\right), 3.28(1 \mathrm{H}, \mathrm{dd}, J=7.4, J=2.8$, $\mathrm{CH}(\mathrm{NH}) \mathrm{CHO}), 1.96\left(3 \mathrm{H}, \mathrm{s}, \mathrm{CH}_{3} \mathrm{CONH}\right), 1.82-1.65\left(2 \mathrm{H}, \mathrm{m}, 2 \times \mathrm{CHCH}_{3}\right), 1.27\left(3 \mathrm{H}, \mathrm{s}, \mathrm{CH}_{3} \mathrm{CO}_{2}\right), 1.22\left(3 \mathrm{H}, \mathrm{s}, \mathrm{CH}_{3} \mathrm{CO}_{2}\right)$, $1.15\left(3 \mathrm{H}, \mathrm{d}, J=6.7, \mathrm{CH}_{3} \mathrm{CHNH}\right), 1.07\left(9 \mathrm{H}, \mathrm{s}, \mathrm{C}\left(\mathrm{CH}_{3}\right)_{3}\right), 0.93\left(3 \mathrm{H}, \mathrm{d}, J=6.7, \mathrm{CHCH}_{3}\right), 0.90\left(3 \mathrm{H}, \mathrm{d}, J=6.7, \mathrm{CHC}_{3}\right) .{ }^{13} \mathbf{C}$ NMR (100.6 MHz, $\left.\mathrm{CDCl}_{3}\right) \delta 169.0(\mathrm{C}), 135.8(\mathrm{CH}), 135.7(\mathrm{CH}), 134.0(\mathrm{C}), 133.8(\mathrm{C}), 129.5(\mathrm{CH}), 129.4(\mathrm{CH}), 127.5$ $(\mathrm{CH}), 127.4(\mathrm{CH}), 100.6(\mathrm{C}), 77.4(\mathrm{CH}), 69.5(\mathrm{CH}), 65.1\left(\mathrm{CH}_{2}\right), 46.6(\mathrm{CH}), 35.4(\mathrm{CH}), 34.2(\mathrm{CH}), 27.0\left(\mathrm{CH}_{3}\right), 25.1$ $\left(\mathrm{CH}_{3}\right), 23.7\left(\mathrm{CH}_{3}\right), 23.5\left(\mathrm{CH}_{3}\right), 19.3(\mathrm{C}), 14.1\left(\mathrm{CH}_{3}\right), 13.1\left(\mathrm{CH}_{3}\right), 12.0\left(\mathrm{CH}_{3}\right)$. HRMS (+FAB): $m / z$ calcd. for $\mathrm{C}_{30} \mathrm{H}_{46} \mathrm{NO}_{4} \mathrm{Si}[\mathrm{M}+\mathrm{H}]^{+}:$512.3196. Found: 512.3178 .

\section{Hydroxy amide 11 .}

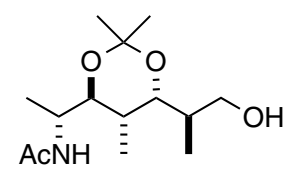

A $1 \mathrm{M}$ solution of TBAF $3 \mathrm{H}_{2} \mathrm{O}$ in THF $(1.4 \mathrm{~mL}, 1.4 \mathrm{mmol})$ was added to a solution of 10 (61.2 $\left.\mathrm{mg}, 0.12 \mathrm{mmol}\right)$ in THF $(0.8 \mathrm{~mL})$ at $0{ }^{\circ} \mathrm{C}$ under $\mathrm{N}_{2}$. The reaction mixture was stirred for $1 \mathrm{~h}$ at $0{ }^{\circ} \mathrm{C}$ and $30 \mathrm{~h}$ at $\mathrm{rt}$.

The solvent was removed and the resulting oil was purified by flash column chromatography (hexanes/EtOAc 90:10), which provided $32.4 \mathrm{mg}(99 \%)$ of $\mathbf{1 1 .}$

White solid. $\boldsymbol{R}_{\boldsymbol{f}}(\mathrm{EtOAc})=0.30 .[\boldsymbol{\alpha}]_{\mathbf{D}}=-3.5\left(c=1.46, \mathrm{CHCl}_{3}\right)$. IR (film): v 3301 (br), 2983, 2937, 1654, 1559, 1459, 1380, 1225, $1022 \mathrm{~cm}^{-1} .{ }^{1} \mathbf{H}$ NMR (400 MHz, $\left.\mathrm{CDCl}_{3}\right) \delta 5.76(1 \mathrm{H}, \mathrm{d}, J=8.4, \mathrm{NH}), 4.17-4.02(1 \mathrm{H}, \mathrm{m}, \mathrm{C} \underline{\mathrm{HNH}}), 3.66-3.50$ $\left(3 \mathrm{H}, \mathrm{m}, \mathrm{C} \underline{\mathrm{HOCHCH}} \underline{\mathrm{H}}_{2} \mathrm{OH}\right), 3.23(1 \mathrm{H}, \mathrm{dd}, J=7.7, J=2.9, \mathrm{CH}(\mathrm{NH}) \mathrm{C} \underline{\mathrm{HO}}), 3.18(1 \mathrm{H}, \mathrm{br} \mathrm{s}, \mathrm{OH}), 1.97(3 \mathrm{H}, \mathrm{s}, \mathrm{C} \underline{\mathrm{H}} 3 \mathrm{CONH})$, 
1.95-1.75 (2H, m, $\left.2 \times \mathrm{CHCH}_{3}\right), 1.39\left(3 \mathrm{H}, \mathrm{s}, \mathrm{CH}_{3} \mathrm{CO}_{2}\right), 1.33\left(3 \mathrm{H}, \mathrm{s}, \mathrm{CH}_{3} \mathrm{CO}_{2}\right), 1.14\left(3 \mathrm{H}, \mathrm{d}, J=6.7, \underline{\mathrm{H}}_{3} \mathrm{CHNH}\right), 0.95$ $\left(3 \mathrm{H}, \mathrm{d}, J=6.7, \mathrm{CHCH}_{3}\right), 0.79\left(3 \mathrm{H}, \mathrm{d}, J=6.7, \mathrm{CHCH}_{3}\right) .{ }^{13} \mathbf{C ~ N M R}\left(100.6 \mathrm{MHz}, \mathrm{CDCl}_{3}\right) \delta 169.0(\mathrm{C}), 100.8(\mathrm{C}), 77.2$ $(\mathrm{CH}), 75.6(\mathrm{CH}), 68.6\left(\mathrm{CH}_{2}\right), 46.3(\mathrm{CH}), 34.9(\mathrm{CH}), 34.4(\mathrm{CH}), 25.0\left(\mathrm{CH}_{3}\right), 23.6\left(\mathrm{CH}_{3}\right), 23.4\left(\mathrm{CH}_{3}\right), 14.1\left(\mathrm{CH}_{3}\right), 12.7$ $\left(\mathrm{CH}_{3}\right), 11.9\left(\mathrm{CH}_{3}\right)$. HRMS (+FAB): $m / z$ calcd. for $\mathrm{C}_{14} \mathrm{H}_{28} \mathrm{NO}_{4}[\mathrm{M}+\mathrm{H}]^{+}: 274.2018$. Found: 274.2024 .

\section{Formyl amide 12.}<smiles>CC(C)NC(C)C1OC(C)(C)OC1C(C)C=O</smiles>

DMSO $(24 \mu \mathrm{L}, 0.34 \mathrm{mmol})$ was added dropwise to a solution of $(\mathrm{COCl})_{2}(15 \mu \mathrm{L}, 0.17 \mathrm{mmol})$ in $\mathrm{CH}_{2} \mathrm{Cl}_{2}(0.35 \mathrm{~mL})$ at -78 ${ }^{\circ} \mathrm{C}$ under $\mathrm{N}_{2}$. The resulting mixture was stirred for $1 \mathrm{~h}$ at $-78{ }^{\circ} \mathrm{C}$ and a solution of $\mathbf{1 1}(31.3 \mathrm{mg}, 0.11 \mathrm{mmol})$ in $\mathrm{CH}_{2} \mathrm{Cl}_{2}$ $(0.60 \mathrm{~mL})$ was added dropwise. The reaction mixture was stirred for $1 \mathrm{~h}$ at $-78{ }^{\circ} \mathrm{C}$, followed by the addition of $\mathrm{Et}_{3} \mathrm{~N}(72$ $\mu \mathrm{L}, 0.51 \mathrm{mmol})$.

The resulting white suspension was allowed to warm to $\mathrm{rt}$ and stirred for $2 \mathrm{~h}$. It was partitioned between $\mathrm{CH}_{2} \mathrm{Cl}_{2}(20 \mathrm{~mL})$ and $\mathrm{H}_{2} \mathrm{O}(20 \mathrm{~mL})$. The aqueous layer was further extracted with $\mathrm{CH}_{2} \mathrm{Cl}_{2}(3 \times 20 \mathrm{~mL})$. The combined organic extracts were washed with $\mathrm{H}_{2} \mathrm{O}(2 \times 10 \mathrm{~mL})$ and brine $(10 \mathrm{~mL})$, dried $\left(\mathrm{Na}_{2} \mathrm{SO}_{4}\right)$, filtered, and concentrated. The residue was filtered through a pad of silica gel (EtOAc) and concentrated to give a colorless oil $(35.7 \mathrm{mg})$ that was taken on to the next step without further purification.

$\boldsymbol{R}_{f}(\mathrm{EtOAc})=0.40 .{ }^{1} \mathbf{H}$ NMR $\left(300 \mathrm{MHz}, \mathrm{CDCl}_{3}\right) \delta 9.68(1 \mathrm{H}, \mathrm{d}, J=2.7, \mathrm{CH}=\mathrm{O}), 5.78(1 \mathrm{H}, \mathrm{d}, J=8.6, \mathrm{NH}), 4.20-4.00$ $(1 \mathrm{H}, \mathrm{m}, \mathrm{NCH}), 3.91(1 \mathrm{H}, \mathrm{dd}, J=10.8, J=4.3, \mathrm{CHO}), 3.35(1 \mathrm{H}, \mathrm{dd}, J=7.6, J=3.1, \mathrm{CHO}), 2.57-2.42(1 \mathrm{H}, \mathrm{m}$, $\mathrm{CHCH}=\mathrm{O}), 1.98\left(3 \mathrm{H}, \mathrm{s}, \mathrm{CH}_{3} \mathrm{CONH}\right), 1.90-1.75\left(1 \mathrm{H}, \mathrm{m}, \mathrm{CHCH}_{3}\right), 1.32\left(3 \mathrm{H}, \mathrm{s}, \mathrm{CH}_{3} \mathrm{CO}_{2}\right), 1.31\left(3 \mathrm{H}, \mathrm{s}, \mathrm{CH}_{3} \mathrm{CO}_{2}\right), 1.16(3 \mathrm{H}$, d, $\left.J=6.7, \mathrm{CHCH}_{3}\right), 0.99\left(3 \mathrm{H}, \mathrm{d}, J=7.0, \mathrm{CHCH}_{3}\right), 0.96\left(3 \mathrm{H}, \mathrm{d}, J=6.7, \mathrm{CHCH}_{3}\right) .{ }^{13} \mathbf{C} \mathbf{N M R}\left(75.4 \mathrm{MHz}, \mathrm{CDCl}_{3}\right) \delta 204.2$, $169.0,100.9,77.2,70.1,46.4,45.8,33.9,24.8,23.4,23.4,14.2,10.0,8.6$. 


\section{Vinyl iodide 2.}<smiles>CC(C)C1OC(C)(C)OC1C(C)C=CI</smiles>

$\mathrm{CrCl}_{2}(105 \mathrm{mg}, 0.81 \mathrm{mmol})$ was added to a $10 \mathrm{~mL}$ round bottom flask and gently flame dried under high vacuum. Upon cooling, the flask was released under argon, charged with THF $(0.9 \mathrm{~mL})$ and the resulting slurry stirred for 10 min at 0 ${ }^{\circ} \mathrm{C}$. Then, a solution of $2(31.0 \mathrm{mg}, 0.11 \mathrm{mmol})$ and $\mathrm{CHI}_{3}(91 \mathrm{mg}, 0.23 \mathrm{mmol})$ in THF $(1.5 \mathrm{~mL})$ was added dropwise via syringe (it was rinsed with $3 \times 0.2 \mathrm{~mL}$ ). The reaction mixture, which turned to a brown-red color, was stirred for $2 \mathrm{~h}$ at 0 ${ }^{\circ} \mathrm{C}$ and overnight at $\mathrm{rt}$ in the dark.

The mixture was diluted with $\mathrm{Et}_{2} \mathrm{O}(65 \mathrm{~mL})$ and washed with saturated $\mathrm{NaHCO}_{3}(2 \times 30 \mathrm{~mL})$. The aqueous layers were extracted with $\mathrm{Et}_{2} \mathrm{O}(3 \times 45 \mathrm{~mL})$ and the combined organic extracts were dried $\left(\mathrm{MgSO}_{4}\right)$, filtered, and concentrated. Purification by flash column chromatography (hexanes/EtOAc 50:50) afforded $27.0 \mathrm{mg}$ (61\% over two steps from 11) of a 95:5 mixture of $E / Z$ olefins 2.

Pale orange oil. $\boldsymbol{R}_{f}$ (hexanes/EtOAc 50:50) $=0.25 .[\alpha]_{\mathbf{D}}=+23.2\left(c=0.75, \mathrm{CHCl}_{3}\right)$. IR (film): v $3282(\mathrm{br}), 2925,2854$, $1654,1559,1542,1459,1378,1225,1023 \mathrm{~cm}^{-1} .{ }^{1} \mathbf{H}$ NMR $\left(400 \mathrm{MHz}, \mathrm{CDCl}_{3}\right) \delta 6.52(1 \mathrm{H}, \mathrm{dd}, J=14.5, J=7.4$, $\mathrm{C} \underline{H}=\mathrm{CHI}), 6.04(1 \mathrm{H}, \mathrm{dd}, J=14.5, J=1.0, \mathrm{CH}=\mathrm{C} \underline{\mathrm{H}}), 5.73(1 \mathrm{H}, \mathrm{br} \mathrm{d}, J=8.4, \mathrm{NH}), 4.10-4.00(1 \mathrm{H}, \mathrm{m}, \mathrm{C} \underline{H N H}), 3.40(1 \mathrm{H}$, dd, $J=10.4, J=4.2$, C $\underline{H O C H C H}=\mathrm{CHI}), 3.30(1 \mathrm{H}, \mathrm{dd}, J=7.5, J=2.9, \mathrm{CH}(\mathrm{NH}) \underline{\mathrm{HO}}), 2.35-2.25(1 \mathrm{H}, \mathrm{m}, \mathrm{C} \underline{\mathrm{HCH}}=\mathrm{CHI})$, $1.97\left(3 \mathrm{H}, \mathrm{s}, \mathrm{C}_{3} \mathrm{CONH}\right), 1.85-1.75(1 \mathrm{H}, \mathrm{m}, \mathrm{OCHC} \underline{\mathrm{HCHO}}), 1.31\left(3 \mathrm{H}, \mathrm{s}, \mathrm{CH}_{3} \mathrm{CO}_{2}\right), 1.30\left(3 \mathrm{H}, \mathrm{s}, \mathrm{CH}_{3} \mathrm{CO}_{2}\right), 1.13(3 \mathrm{H}, \mathrm{d}, J$ $\left.=6.7, \mathrm{C}_{3} \mathrm{CHNH}\right), 0.95\left(3 \mathrm{H}, \mathrm{d}, J=6.7, \mathrm{CH}\left(\mathrm{CH}_{3}\right) \mathrm{CH}=\mathrm{CHI}\right), 0.92\left(3 \mathrm{H}, \mathrm{d}, J=6.7, \mathrm{OCHCH}\left(\mathrm{CH}_{3}\right) \mathrm{CHO}\right) .{ }^{13} \mathbf{C} \mathbf{~ N M R}(100.6$ $\left.\mathrm{MHz}, \mathrm{CDCl}_{3}\right) \delta 169.0(\mathrm{C}), 149.2(\mathrm{CH}), 100.9(\mathrm{C}), 77.3(\mathrm{CH}), 74.6(\mathrm{CH}), 72.4(\mathrm{CH}), 46.4(\mathrm{CH}), 39.9(\mathrm{CH}), 34.4(\mathrm{CH})$, $24.7\left(\mathrm{CH}_{3}\right), 23.6\left(\mathrm{CH}_{3}\right), 23.5\left(\mathrm{CH}_{3}\right), 15.2\left(\mathrm{CH}_{3}\right), 14.1\left(\mathrm{CH}_{3}\right), 11.8\left(\mathrm{CH}_{3}\right)$. HRMS (+FAB): $m / z$ calcd. for $\mathrm{C}_{15} \mathrm{H}_{27} \mathrm{NO}_{3} \mathrm{I}$ $[\mathrm{M}+\mathrm{H}]^{+}:$396.1036. Found: 396.1022 . 


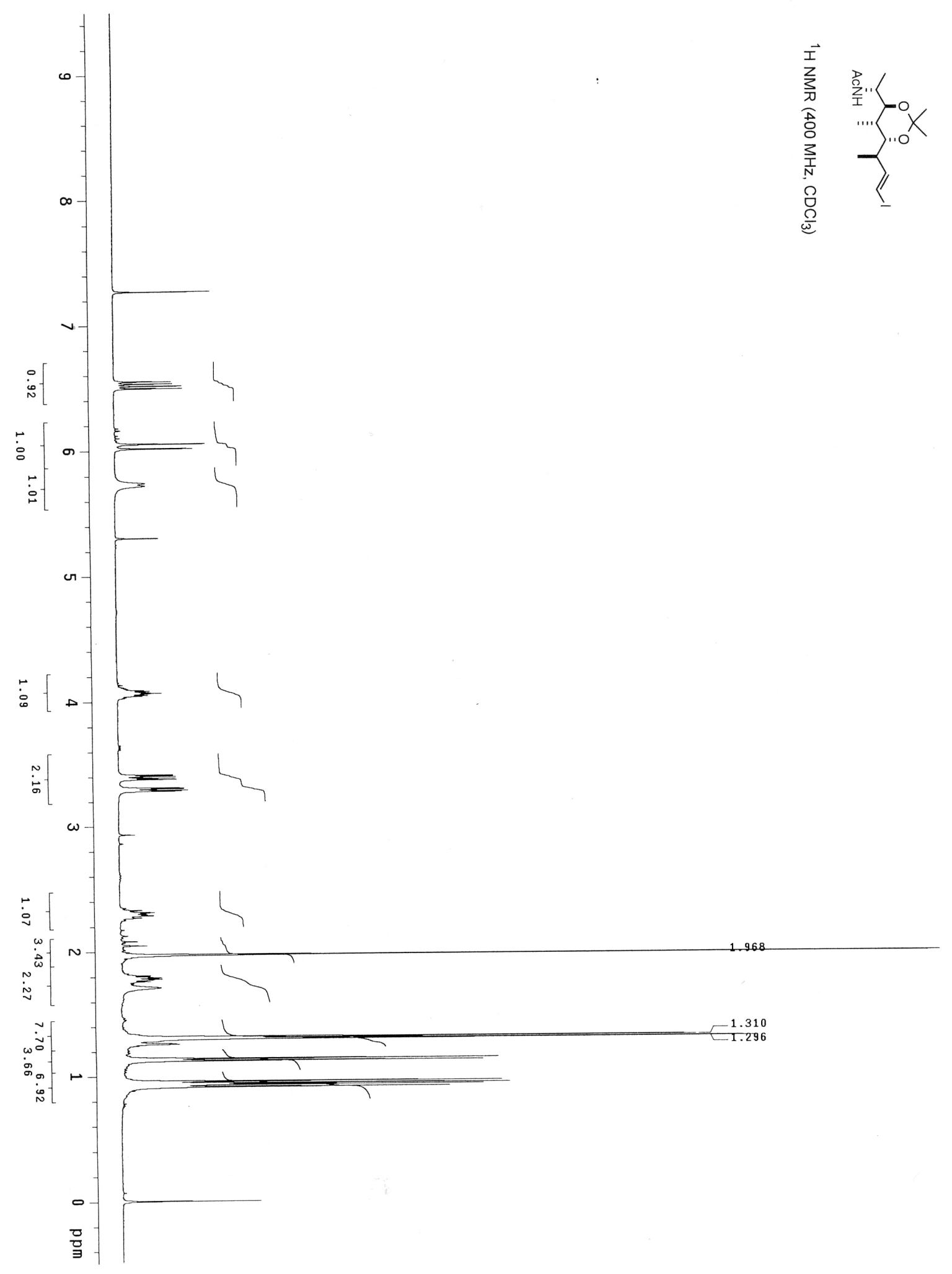


Supporting information for Studies Directed Toward the Construction of the Polypropionate Fragment of Superstolide A Joan G. Solsona, Pedro Romea, Fèlix Urpí

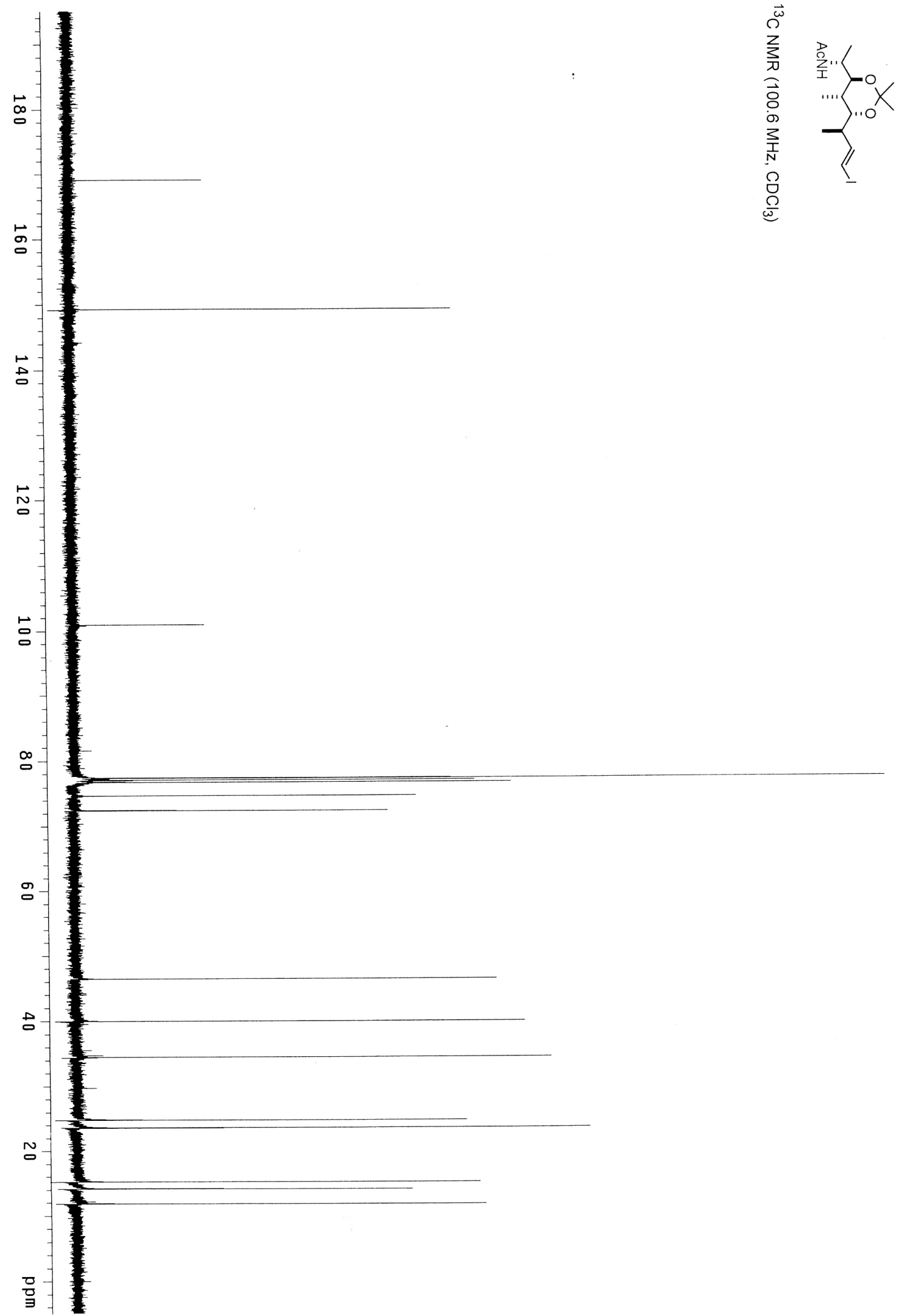




\section{9. (2R,3R,4S,5S,6R)-6-Azido-3,5-isopropylidenedioxy-2,4-dimethyl-1-heptanol (14).}<smiles>CC(CO)[C@@H]1OC(C)(C)O[C@H](C(C)CO)[C@H]1C</smiles>

A $1 \mathrm{M}$ solution of TBAF $3 \mathrm{H}_{2} \mathrm{O}$ in THF $(3 \mathrm{~mL}, 3 \mathrm{mmol})$ was added to a solution of 4 (123 $\left.\mathrm{mg}, 0.25 \mathrm{mmol}\right)$ in THF (1.7 $\mathrm{mL}$ ) at $0{ }^{\circ} \mathrm{C}$ under $\mathrm{N}_{2}$. The reaction mixture was stirred for $1 \mathrm{~h}$ at $0{ }^{\circ} \mathrm{C}$ and overnight at $\mathrm{rt}$.

It was diluted with $\mathrm{Et}_{2} \mathrm{O}(100 \mathrm{~mL})$ and washed with a $10 \%$ aqueous solution of $\mathrm{KHSO}_{4} 10 \%(40 \mathrm{~mL})$, saturated $\mathrm{NaHCO}_{3}$ $(40 \mathrm{~mL})$ and $\mathrm{H}_{2} \mathrm{O}(40 \mathrm{~mL})$. The organic layer was dried $\left(\mathrm{MgSO}_{4}\right)$, filtered, and concentrated. Purification by flash column chromatography (hexanes/EtOAc 90:10) afforded $58 \mathrm{mg}(91 \%)$ of $\mathbf{1 4 .}$

Colorless oil. $\boldsymbol{R}_{\boldsymbol{f}}$ (hexanes/EtOAc 85:15) =0.17. $[\boldsymbol{\alpha}]_{\mathbf{D}}=-58.1\left(c=1.13, \mathrm{CHCl}_{3}\right)$. IR (film): v 3350 (br), 2980, 2940, 2120, 1380, 1240, $1030 \mathrm{~cm}^{-1} .{ }^{1} \mathbf{H}$ NMR $\left(400 \mathrm{MHz}, \mathrm{CDCl}_{3}\right) \delta 3.67\left(1 \mathrm{H}, \mathrm{dd}, J=10.7, J=4.0, \underline{\mathrm{H}}_{\mathrm{x}} \mathrm{H}_{\mathrm{y}} \mathrm{OH}\right), 3.63(1 \mathrm{H}, \mathrm{dd}, J$ $\left.=10.7, J=7.9, \mathrm{CH}_{\mathrm{x}} \underline{\mathrm{H}}_{\mathrm{y}} \mathrm{OH}\right), 3.57-3.52\left(1 \mathrm{H}, \mathrm{m}, \mathrm{CH}\left(\mathrm{N}_{3}\right) \mathrm{CHO}\right), 3.50\left(1 \mathrm{H}, \mathrm{qd}, J=6.7, J=4.0, \mathrm{CHN}_{3}\right), 3.40(1 \mathrm{H}, \mathrm{dd}, J=6.8$, $\left.J=4.0, \mathrm{OC}_{\mathbf{H C H C H}} \mathrm{OH}\right), 3.10(1 \mathrm{H}$, br s, OH$), 1.96-1.82\left(2 \mathrm{H}, \mathrm{m}, 2 \times \mathrm{CHCH}_{3}\right), 1.40\left(3 \mathrm{H}, \mathrm{s}, \mathrm{CH}_{3} \mathrm{CO}_{2}\right), 1.37(3 \mathrm{H}, \mathrm{s}$, $\left.\mathrm{CH}_{3} \mathrm{CO}_{2}\right), 1.26\left(3 \mathrm{H}, \mathrm{d}, J=6.7, \mathrm{CH}_{3} \mathrm{CHN}_{3}\right), 0.96\left(3 \mathrm{H}, \mathrm{d}, J=6.7, \mathrm{CHCH}_{3}\right), 0.80\left(3 \mathrm{H}, \mathrm{d}, J=6.8, \mathrm{CHCH}_{3}\right) .{ }^{13} \mathbf{C ~ N M R}(100.6$ $\left.\mathrm{MHz}, \mathrm{CDCl}_{3}\right) \delta 100.9(\mathrm{C}), 77.7(\mathrm{CH}), 75.6(\mathrm{CH}), 68.6\left(\mathrm{CH}_{2}\right), 59.4(\mathrm{CH}), 34.9(\mathrm{CH}), 34.2(\mathrm{CH}), 25.3\left(\mathrm{CH}_{3}\right), 23.5\left(\mathrm{CH}_{3}\right)$, $14.5\left(\mathrm{CH}_{3}\right), 12.6\left(\mathrm{CH}_{3}\right), 12.5\left(\mathrm{CH}_{3}\right)$. HRMS (+FAB): $m / z$ calcd. for $\mathrm{C}_{12} \mathrm{H}_{24} \mathrm{~N}_{3} \mathrm{O}_{3}[\mathrm{M}+\mathrm{H}]^{+}: 258.1818$. Found: 258.1828.

\section{Azido sulfone 15.}

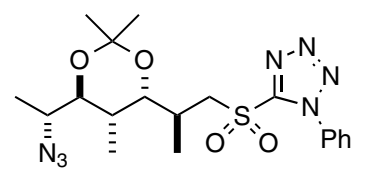

DEAD $(51 \mu \mathrm{L}, 033 \mathrm{mmol})$ was added to a solution of $\mathrm{Ph}_{3} \mathrm{P}(75 \mathrm{mg}, 0.29 \mathrm{mmol})$ and 1-phenyl-1H-tetrazole-5-thiol (60 $\mathrm{mg}, 0.33 \mathrm{mmol})$ in THF $(1.5 \mathrm{~mL})$ at $0{ }^{\circ} \mathrm{C}$ under $\mathrm{N}_{2}$. It was stirred for $10 \mathrm{~min}$ and a solution of $14(56 \mathrm{mg}, 0.22 \mathrm{mmol})$ in THF $(0.55 \mathrm{~mL})$ was added dropwise (it was rinsed with $3 \times 0.25 \mathrm{~mL}$ of THF). The resulting bright yellow solution was stirred for $15 \mathrm{~min}$ at $0{ }^{\circ} \mathrm{C}$ and $4 \mathrm{~h}$ at rt. It was finally diluted with $\mathrm{EtOH}(4.6 \mathrm{~mL})$ and cooled to $0{ }^{\circ} \mathrm{C}$.

In a separate flask, $30 \%$ aq $\mathrm{H}_{2} \mathrm{O}_{2}(0.9 \mathrm{~mL}, 8.7 \mathrm{mmol})$ and $\left(\mathrm{NH}_{4}\right)_{2} \mathrm{MoO}_{4}(136 \mathrm{mg}, 0.11 \mathrm{mmol})$ were mixed, producing a bright yellow solution that was added dropwise to the reaction mixture.

The resulting yellow suspension was stirred for $10 \mathrm{~min}$ at $0{ }^{\circ} \mathrm{C}$ and one day at $\mathrm{rt}$ and partitioned between $\mathrm{CH}_{2} \mathrm{Cl}_{2}(35 \mathrm{~mL})$ and $\mathrm{H}_{2} \mathrm{O}(15 \mathrm{~mL})$. The layers were separated and the aqueous one was extracted with $\mathrm{CH}_{2} \mathrm{Cl}_{2}(3 \times 20 \mathrm{~mL})$. The combined organic extracts were washed with $\mathrm{H}_{2} \mathrm{O}(20 \mathrm{~mL})$ and brine $(20 \mathrm{~mL})$, dried $\left(\mathrm{MgSO}_{4}\right)$, filtered, and concentrated.

Purification by flash chromatography (hexanes/EtOAc 85:15) provided $82 \mathrm{mg}$ (84\%) of 15.

Colorless wax. $\boldsymbol{R}_{\boldsymbol{f}}\left(\right.$ hexanes/EtOAc 75:25) $=0.52 .[\alpha]_{\mathbf{D}}=-22.7\left(c=1.11, \mathrm{CHCl}_{3}\right)$. IR (film): $v 2985,2939,2105,1499$, 
1383, 1343, 1225, 1154, $1026 \mathrm{~cm}^{-1} .{ }^{1} \mathbf{H}$ NMR (400 MHz, $\left.\mathrm{CDCl}_{3}\right) \delta 7.70-7.50(5 \mathrm{H}, \mathrm{m}, \mathrm{ArH}), 4.18(1 \mathrm{H}, \mathrm{dd}, J=14.5, J=$ 2.5, $\left.\mathrm{C}_{\mathrm{x}} \mathrm{H}_{\mathrm{y}} \mathrm{SO}_{2}\right), 3.56-3.44\left(3 \mathrm{H}, \mathrm{m}, \mathrm{CH}_{\mathrm{x}} \underline{\mathrm{H}}_{\mathrm{y}} \mathrm{SO}_{2}, \mathrm{CHN}_{3}\right.$ and $\left.\mathrm{CHOCHCH}_{2} \mathrm{SO}_{2}\right), 3.36(1 \mathrm{H}, \mathrm{dd}, J=6.6, J=4.1$, $\left.\mathrm{CH}\left(\mathrm{N}_{3}\right) \mathrm{CHO}\right), 2.48-2.32\left(1 \mathrm{H}, \mathrm{m}, \mathrm{CHCHSO}_{2}\right), 2.02-1.92(1 \mathrm{H}, \mathrm{m}, \mathrm{CHOC} \underline{\mathrm{HCHO}}), 1.33\left(3 \mathrm{H}, \mathrm{s}, \mathrm{CH}_{3} \mathrm{CO}_{2}\right), 1.32(3 \mathrm{H}, \mathrm{s}$, $\left.\mathrm{CH}_{3} \mathrm{CO}_{2}\right), 1.24\left(3 \mathrm{H}, \mathrm{d}, J=6.7, \mathrm{CH}_{3} \mathrm{CHN}_{3}\right), 1.13\left(3 \mathrm{H}, \mathrm{d}, J=6.7, \mathrm{CH}\left(\mathrm{CH}_{3}\right) \mathrm{CH}_{2} \mathrm{SO}_{2}\right), 0.93(3 \mathrm{H}, \mathrm{d}, J=6.8$, $\left.\mathrm{OHCCH}\left(\mathrm{CH}_{3}\right) \mathrm{CHO}\right) .{ }^{13} \mathrm{C}$ NMR $\left(100.6 \mathrm{MHz}, \mathrm{CDCl}_{3}\right) \delta 154.2(\mathrm{C}), 133.1(\mathrm{C}), 131.4(\mathrm{CH}), 129.6(\mathrm{CH}), 125.3(\mathrm{CH}), 101.2$ (C), $77.7(\mathrm{CH}), 71.6(\mathrm{CH}), 59.5(\mathrm{CH}), 58.8\left(\mathrm{CH}_{2}\right), 33.6(\mathrm{CH}), 29.0(\mathrm{CH}), 25.1\left(\mathrm{CH}_{3}\right), 23.3\left(\mathrm{CH}_{3}\right), 15.2\left(\mathrm{CH}_{3}\right), 14.6\left(\mathrm{CH}_{3}\right)$, $12.3\left(\mathrm{CH}_{3}\right)$. HRMS (+FAB): $m / z$ calcd. for $\mathrm{C}_{19} \mathrm{H}_{28} \mathrm{~N}_{7} \mathrm{O}_{4} \mathrm{~S}[\mathrm{M}+\mathrm{H}]^{+}: 450.1923$. Found: 450.1921 .

\section{Azido diene 16.}

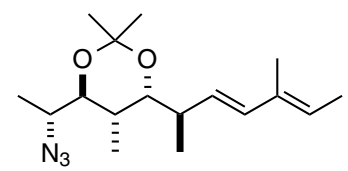

A $1 \mathrm{M}$ solution of LiHMDS in THF $(0.36 \mathrm{~mL}, 0.36 \mathrm{mmol})$ was added dropwise to a solution of $15(81 \mathrm{mg}, 0.18 \mathrm{mmol})$ and (E)-2-methyl-2-butenal $(0.22 \mathrm{~mL}, 0.22 \mathrm{mmol})$ in 1,2-dimethoxyethane $(4.2 \mathrm{~mL})$ at $-65{ }^{\circ} \mathrm{C}$ under $\mathrm{N}_{2}$. The resulting yellow solution was stirred at $-65^{\circ} \mathrm{C}$ for $6 \mathrm{~h}$, allowed to warm very slowly, and stirred for an additional hour at $\mathrm{rt}$.

The reaction was quenched by addition of saturated $\mathrm{NH}_{4} \mathrm{Cl}(5 \mathrm{~mL})$ and partitioned between $\mathrm{Et}_{2} \mathrm{O}(120 \mathrm{~mL})$ and saturated $\mathrm{NH}_{4} \mathrm{Cl}(60 \mathrm{~mL})$. The aqueous layer was extracted with $\mathrm{Et}_{2} \mathrm{O}(3 \times 80 \mathrm{~mL})$ and the combined organic extracts were washed with brine $(80 \mathrm{~mL})$, dried $\left(\mathrm{MgSO}_{4}\right)$, filtered, and concentrated. Purification by flash column chromatography (hexanes/EtOAc 97:3) afforded $40 \mathrm{mg}(72 \%)$ of $>94: 6$ mixture of $E, E$ - and $Z, E-16$ dienes according to the ${ }^{1} \mathrm{H}$ NMR (400 $\mathrm{MHz}$ ).

Colorless oil. $\boldsymbol{R}_{\boldsymbol{f}}$ (hexanes/EtOAc 95:5) = 0.43. $[\boldsymbol{\alpha}]_{\mathbf{D}}=-44.5\left(c=1.72, \mathrm{CH}_{2} \mathrm{Cl}_{2}\right)$. IR (film): v 2985, 2937, 2101, 1457, $1380,1263,1225,1183,1027 \mathrm{~cm}^{-1} .{ }^{1} \mathbf{H}$ NMR $\left(400 \mathrm{MHz}, \mathrm{CDCl}_{3}\right) \delta 6.09(1 \mathrm{H}, \mathrm{d}, J=15.8, \mathrm{CHCH}=\mathrm{CH}), 5.57(1 \mathrm{H}, \mathrm{dd}, J=$ 15.8, $J=7.0, \mathrm{CHC} \underline{\mathrm{H}}=\mathrm{CH}), 5.50-5.40\left(1 \mathrm{H}, \mathrm{m}, \mathrm{C}=\mathrm{CHCH}_{3}\right), 3.55-3.40\left(2 \mathrm{H}, \mathrm{m}, \mathrm{C} \underline{\mathrm{HOCHCH}}=\mathrm{CH}\right.$ and $\left.\mathrm{CHN}_{3}\right), 3.37(1 \mathrm{H}, \mathrm{dd}$, $\left.J=6.8, J=4.0, \mathrm{CH}\left(\mathrm{N}_{3}\right) \mathrm{C} \underline{H O}\right), 2.35-2.25(1 \mathrm{H}, \mathrm{m}, \mathrm{CHOC} \underline{\mathrm{HCH}}=\mathrm{CH}), 1.91(1 \mathrm{H}$, quintetd, $J=6.7, J=4.0, \mathrm{OCHC} \underline{\mathrm{HCHO}})$, $1.71\left(3 \mathrm{H}, \mathrm{s},\left(\mathrm{CH}_{3}\right) \mathrm{C}=\mathrm{CHCH}_{3}\right), 1.70\left(3 \mathrm{H}, \mathrm{d}, J \approx 6.0,\left(\mathrm{CH}_{3}\right) \mathrm{C}=\mathrm{CHCH}_{3}\right), 1.35\left(3 \mathrm{H}, \mathrm{s}, \mathrm{CH}_{3} \mathrm{CO}_{2}\right), 1.29\left(3 \mathrm{H}, \mathrm{s}, \mathrm{CH}_{3} \mathrm{CO}_{2}\right), 1.25$ $\left(3 \mathrm{H}, \mathrm{d}, J=6.8, \mathrm{CH}_{3} \mathrm{CHN}_{3}\right), 0.96\left(3 \mathrm{H}, \mathrm{d}, J=6.7, \mathrm{CHC}_{3}\right), 0.95(3 \mathrm{H}, \mathrm{d}, \mathrm{J}=6.7, \mathrm{CHCH} 3) .{ }^{13} \mathbf{C ~ N M R}\left(100.6 \mathrm{MHz}, \mathrm{CDCl}_{3}\right)$ $\delta 134.5(\mathrm{C}), 133.8(\mathrm{CH}), 130.2(\mathrm{CH}), 124.6(\mathrm{CH}), 100.9(\mathrm{C}), 77.9(\mathrm{CH}), 73.6(\mathrm{CH}), 59.6(\mathrm{CH}), 35.9(\mathrm{CH}), 34.4(\mathrm{CH})$, $24.9\left(\mathrm{CH}_{3}\right), 23.6\left(\mathrm{CH}_{3}\right), 16.1\left(\mathrm{CH}_{3}\right), 14.5\left(\mathrm{CH}_{3}\right), 13.7\left(\mathrm{CH}_{3}\right), 12.7\left(\mathrm{CH}_{3}\right), 12.0\left(\mathrm{CH}_{3}\right)$. HRMS (+FAB): $m / z$ calcd. for $\mathrm{C}_{17} \mathrm{H}_{30} \mathrm{~N}_{3} \mathrm{O}_{2}[\mathrm{M}+\mathrm{H}]^{+}:$308.2338. Found: 308.2343. 


\section{Amido diene 17.}<smiles>C/C=C(C)/C=C/C(C)[C@H]1OC(C)(C)O[C@H](C(C)N)[C@H]1C</smiles>

A $1 \mathrm{M}$ solution of $\mathrm{Me}_{3} \mathrm{P}$ in toluene $(0.12 \mathrm{~mL}, 0.12 \mathrm{mmol})$ was added dropwise to a solution of $\mathbf{1 6}(30.8 \mathrm{mg}, 0.10 \mathrm{mmol})$ in benzene $(0.5 \mathrm{~mL})$ under $\mathrm{N}_{2}$. The reaction mixture was stirred for $2 \mathrm{~h}$ at $\mathrm{rt}$ and cooled at $0{ }^{\circ} \mathrm{C}$. Then, a $1 \mathrm{M}$ solution of $\mathrm{AcCl}$ in benzene $(0.12 \mathrm{~mL}, 0.12 \mathrm{mmol})$ was added slowly and the resulting mixture was allowed to stir for 5 min at $0{ }^{\circ} \mathrm{C}$ and $5 \mathrm{~h}$ at $\mathrm{rt}$.

The reaction was quenched by addition of $\mathrm{H}_{2} \mathrm{O}(5 \mathrm{~mL})$, stirred for $15 \mathrm{~min}$ and extracted with $\mathrm{CH}_{2} \mathrm{Cl}_{2}(3 \times 25 \mathrm{~mL})$. The combined organic extracts were dried $\left(\mathrm{Na}_{2} \mathrm{SO}_{4}\right)$, filtered, and concentrated. Purification by flash column chromatography (hexanes/EtOAc 35:65) afforded $20.7 \mathrm{mg}$ (64\%) of 17.

White solid. $\boldsymbol{R}_{f}$ (hexanes/EtOAc 35:65) = 0.32. $[\boldsymbol{\alpha}]_{\mathbf{D}}=+17.1\left(c=1.10, \mathrm{CH}_{2} \mathrm{Cl}_{2}\right)$. IR (film): $v 3286(\mathrm{br}), 2985,2935,2101$, 1648, 1551, 1459, 1378, 1225, 1183, $1023 \mathrm{~cm}^{-1} .{ }^{1} \mathbf{H}$ NMR $\left(400 \mathrm{MHz}, \mathrm{CDCl}_{3}\right) \delta 6.08(1 \mathrm{H}, \mathrm{d}, J=15.8, \mathrm{CHCH}=\mathrm{C} \underline{H})$, 5.80-5.70 (1H, m, NH), $5.56(1 \mathrm{H}, \mathrm{dd}, J=15.8, J=7.0, \mathrm{CHC} \underline{\mathrm{H}}=\mathrm{CH}), 5.50-5.40\left(1 \mathrm{H}, \mathrm{m}, \mathrm{C}=\mathrm{C}^{-} \mathrm{HCH}_{3}\right), 4.10-4.00(1 \mathrm{H}, \mathrm{m}$, $\mathrm{NHC} \underline{\mathrm{H}}), 3.40(1 \mathrm{H}, \mathrm{dd}, J=10.5, J=4.2, \mathrm{C} \underline{\mathrm{HOCHCH}}=\mathrm{CH}), 3.30(1 \mathrm{H}, \mathrm{dd}, J=7.5, J=2.8, \mathrm{CH}(\mathrm{NH}) \mathrm{C} \underline{\mathrm{HO}}), 2.35-2.25(1 \mathrm{H}$, $\mathrm{m}, \mathrm{CHOC} \underline{\mathrm{HCH}}=\mathrm{CH}), 1.97\left(3 \mathrm{H}, \mathrm{s}, \underline{\mathrm{C}}_{3} \mathrm{CONH}\right), 1.85-1.75(1 \mathrm{H}, \mathrm{m}, \mathrm{OCHC} \underline{\mathrm{HCHO}}), 1.71\left(3 \mathrm{H}, \mathrm{s},\left(\mathrm{C}_{3}\right) \mathrm{C}=\mathrm{CHCH}_{3}\right), 1.70$ $\left(3 \mathrm{H}, \mathrm{d}, J \approx 6.0,\left(\mathrm{CH}_{3}\right) \mathrm{C}=\mathrm{CHCH}_{3}\right), 1.31\left(3 \mathrm{H}, \mathrm{s}, \mathrm{CH}_{3} \mathrm{CO}_{2}\right), 1.28\left(3 \mathrm{H}, \mathrm{s}, \mathrm{CH}_{3} \mathrm{CO}_{2}\right), 1.14\left(3 \mathrm{H}, \mathrm{d}, J=6.7, \mathrm{C}_{3} \mathrm{CHNH}\right), 0.95$ $\left(3 \mathrm{H}, \mathrm{d}, J=6.7, \mathrm{CHC}_{3}\right), 0.94\left(3 \mathrm{H}, \mathrm{d}, J=6.6, \mathrm{CHC}_{3}\right) .{ }^{13} \mathbf{C ~ N M R}\left(100.6 \mathrm{MHz}, \mathrm{CDCl}_{3}\right) \delta 169.0(\mathrm{C}), 134.5(\mathrm{C}), 133.7$ $(\mathrm{CH}), 130.2(\mathrm{CH}), 124.6(\mathrm{CH}), 100.8(\mathrm{C}), 77.4(\mathrm{CH}), 73.5(\mathrm{CH}), 46.5(\mathrm{CH}), 36.0(\mathrm{CH}), 34.6(\mathrm{CH}), 24.7\left(\mathrm{CH}_{3}\right), 23.7$ $\left(\mathrm{CH}_{3}\right), 23.5\left(\mathrm{CH}_{3}\right), 16.2\left(\mathrm{CH}_{3}\right), 14.1\left(\mathrm{CH}_{3}\right), 13.7\left(\mathrm{CH}_{3}\right), 12.0\left(\mathrm{CH}_{3}\right), 11.9\left(\mathrm{CH}_{3}\right)$. HRMS $(+\mathrm{FAB}): \mathrm{m} / z$ calcd. for $\mathrm{C}_{19} \mathrm{H}_{34} \mathrm{NO}_{3}[\mathrm{M}+\mathrm{H}]^{+}:$324.2539. Found: 324.2537 . 
Supporting information for Studies Directed Toward the Construction of the Polypropionate Fragment of Superstolide A Joan G. Solsona, Pedro Romea, Fèlix Urpí

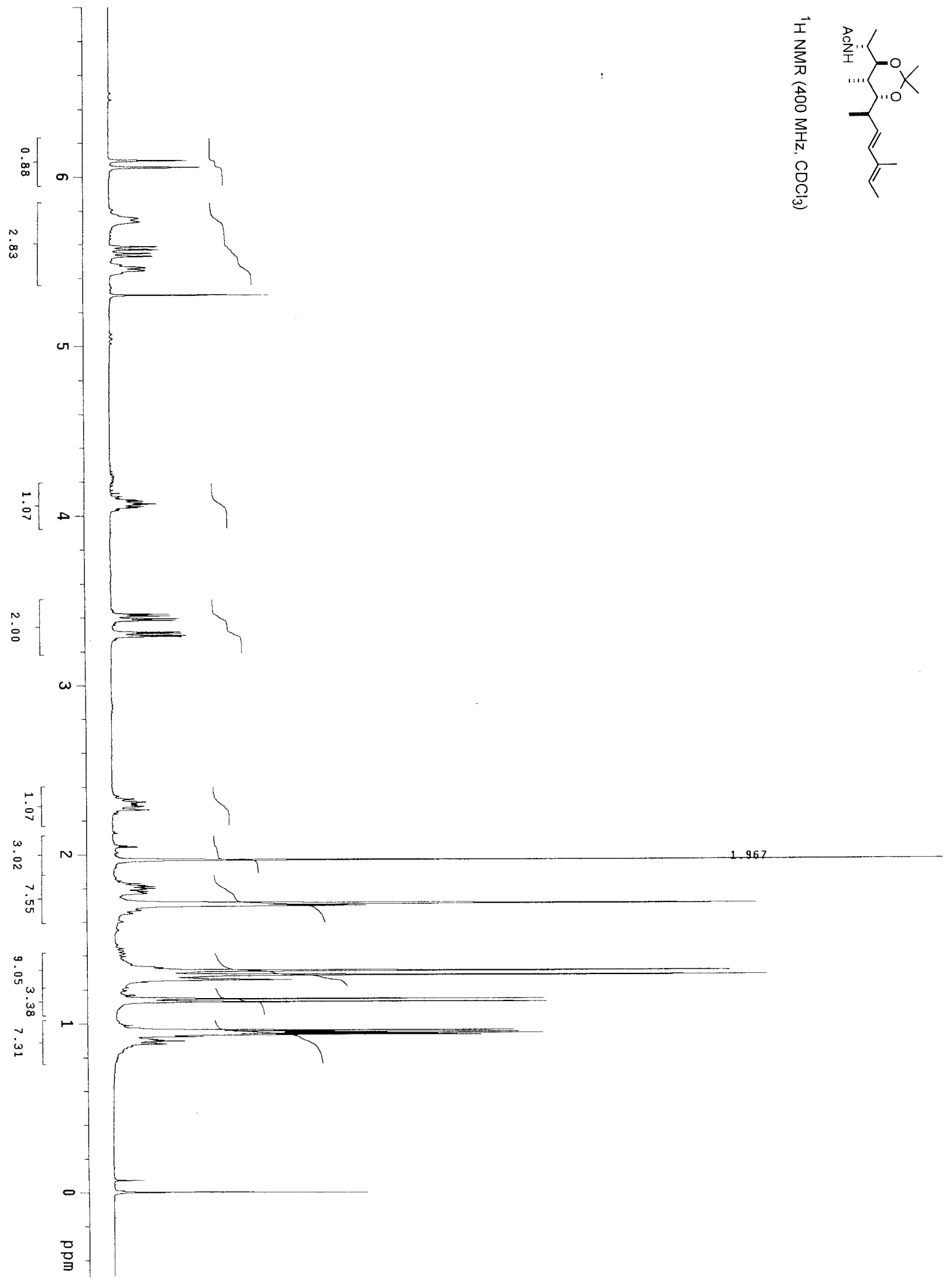




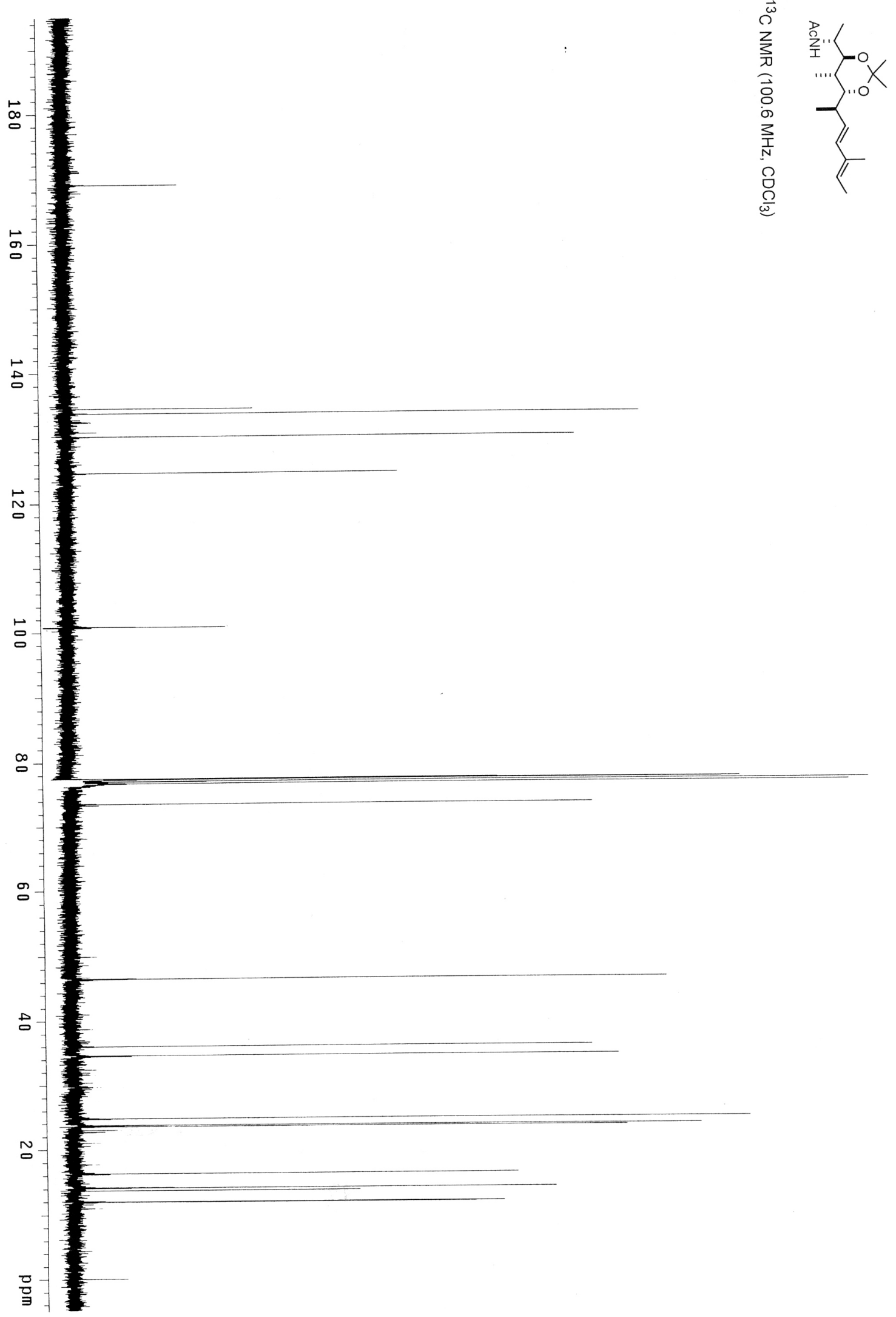




\section{Correlation of Stereochemistry}

Trihydroxy amide 13.<smiles>CC(CO)C(O)C(O)C(C)C(O)C(C)C(N)=O</smiles>

A mixture of $11(17.7 \mathrm{mg}, 60 \mathrm{mmol})$ and Amberlyst $15(35 \mathrm{mg}, 200 \% \mathrm{w} / \mathrm{w})$ in $\mathrm{CH}_{3} \mathrm{OH}(1.8 \mathrm{~mL})$ was shaken for three days at rt. It was filtered and concentrated. Purification of the residue by flash column chromatography $\left(\mathrm{CH}_{2} \mathrm{Cl}_{2} / \mathrm{CH}_{3} \mathrm{OH}\right.$ 90:10) afforded $12.1 \mathrm{mg}(80 \%)$ of $\mathbf{1 3 .}$

White solid. $\boldsymbol{R}_{\boldsymbol{f}}\left(\mathrm{CH}_{2} \mathrm{Cl}_{2} / \mathrm{CH}_{3} \mathrm{OH} 90: 10\right)=0.20$. $[\boldsymbol{\alpha}]_{\mathbf{D}}=+32.4\left(c=0.85, \mathrm{CH}_{3} \mathrm{OH}\right)$. $[\boldsymbol{\alpha}]_{\mathbf{D}}=+25.4\left(c=0.13, \mathrm{CH}_{3} \mathrm{OH}\right)$. IR (KBr): v 3311 (br), 2971, 2931, 1654, 1559, 1542, 1457, 1376, $972 \mathrm{~cm}^{-1} .{ }^{1} \mathbf{H}$ NMR (400 MHz, CD $\left.\mathrm{OD}\right) \delta 4.12-4.04$ $(1 \mathrm{H}, \mathrm{m}, \mathrm{C} \underline{\mathrm{HNH}}), 3.83\left(1 \mathrm{H}, \mathrm{dd}, J=9.9, J=1.7, \underline{\mathrm{CHOCHCH}}_{2} \mathrm{OH}\right), 3.74\left(1 \mathrm{H}, \mathrm{dd}, J=10.6, J=5.3, \mathrm{C}_{\mathrm{x}} \mathrm{H}_{\mathrm{y}} \mathrm{OH}\right), 3.54-3.48$ $\left(2 \mathrm{H}, \mathrm{m}, \mathrm{CH}(\mathrm{NH}) \mathrm{C} \underline{\mathrm{HO}}\right.$ and $\left.\mathrm{CH}_{\mathrm{x}} \underline{\mathrm{H}}_{\mathrm{y}} \mathrm{OH}\right), 1.93\left(3 \mathrm{H}, \mathrm{s}, \mathrm{C}_{3} \mathrm{CONH}\right), 1.80-1.65\left(2 \mathrm{H}, \mathrm{m}, 2 \times \mathrm{C}_{\mathbf{H}} \mathrm{CH}_{3}\right), 1.12(3 \mathrm{H}, \mathrm{d}, J=6.7$, $\left.\mathrm{CH}_{3} \mathrm{CHNH}\right), 0.93\left(3 \mathrm{H}, \mathrm{d}, J=7.0, \mathrm{CHCH}_{3}\right), 0.81\left(3 \mathrm{H}, \mathrm{d}, J=6.8, \mathrm{CHCH}_{3}\right) .{ }^{13} \mathbf{C} \mathbf{N M R}\left(100.6 \mathrm{MHz}, \mathrm{CD}_{3} \mathrm{OD}\right) \delta 172.1,77.3$, $74.3,67.2,48.2,39.5,37.2,22.7,14.9,13.7,9.7$. 
Supporting information for Studies Directed Toward the Construction of the Polypropionate Fragment of Superstolide A Joan G. Solsona, Pedro Romea, Fèlix Urpí

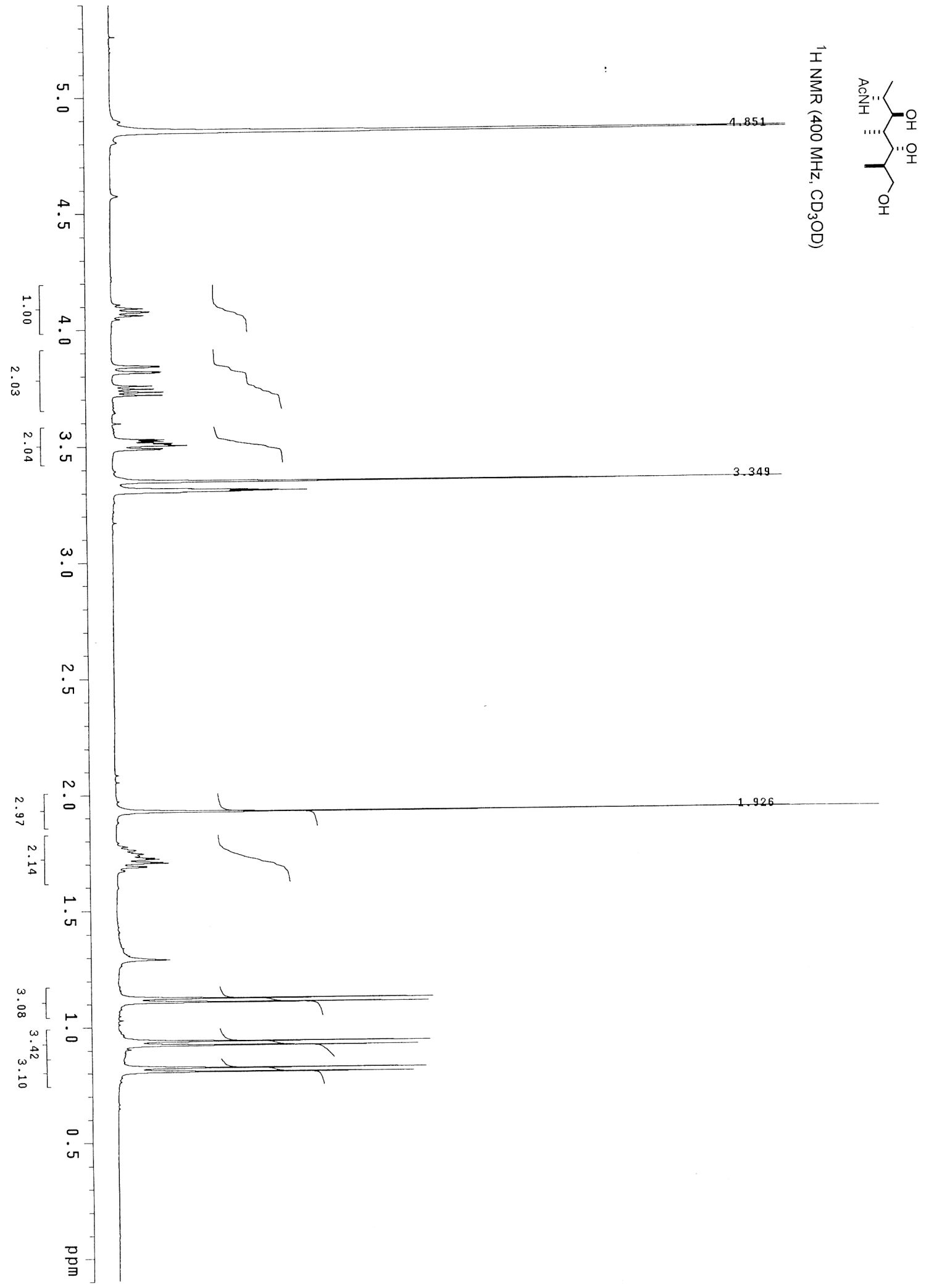




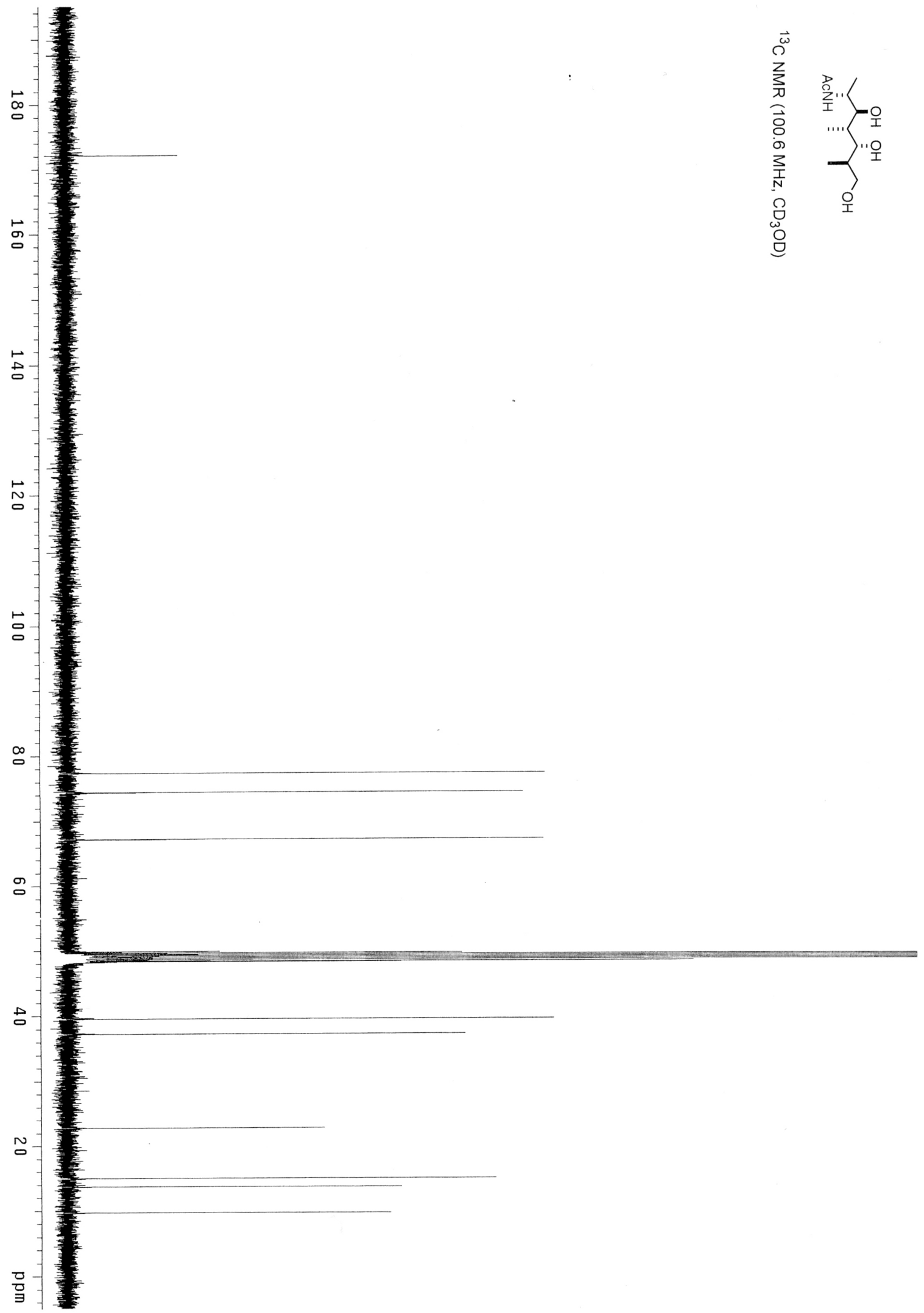

